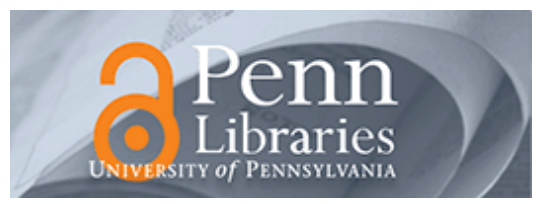

University of Pennsylvania

ScholarlyCommons

Operations, Information and Decisions Papers

Wharton Faculty Research

$1-2005$

\title{
Optimal Product Launch Times in a Duopoly: Balancing Life-Cycle Revenues With Product Cost
}

\author{
Sergei Savin \\ University of Pennsylvania \\ Christian Terwiesch \\ University of Pennsylvania
}

Follow this and additional works at: https://repository.upenn.edu/oid_papers

Part of the Other Business Commons, Other Economics Commons, and the Sales and Merchandising Commons

\section{Recommended Citation}

Savin, S., \& Terwiesch, C. (2005). Optimal Product Launch Times in a Duopoly: Balancing Life-Cycle Revenues With Product Cost. Operations Research, 53 (1), 26-47. http://dx.doi.org/10.1287/

opre.1040.0157

At the time of publication, author Sergei Savin was affiliated with the Columbia University, New York. Currently (July 2016), he is a faculty member in the Operation, Information and Decision amking Department of the Wharton

School at the University of Pennsylvania.

This paper is posted at ScholarlyCommons. https://repository.upenn.edu/oid_papers/122

For more information, please contact repository@pobox.upenn.edu. 


\title{
Optimal Product Launch Times in a Duopoly: Balancing Life-Cycle Revenues With Product Cost
}

\begin{abstract}
We present a model describing the demand dynamics of two new products competing for a limited target market. The demand trajectories of the two products are driven by a market saturation effect and an imitation effect reflecting the product experience of previous adopters. In this general setting, we provide analytical results for the sales trajectories and life-cycle sales of the competing products. We use these results to study the impact of launch time on overall life-cycle sales. We consider the perspective of one of the competing products and model the trade-off between the lost revenues resulting from a delayed launch and the lower unit-production costs. We find that the profit-maximizing launch time exhibits a counterintuitive behavior. In particular, we show that a firm facing a launch time delay from a competing product might benefit from accelerating its own product launch, as opposed to using the softened competitive situation to further improve its cost position. We identify conditions under which a marginal cost-benefit analysis leads to suboptimal launch-time decisions. Finally, we analyze the Nash equilibrium in launch-time decisions of the two competing products.
\end{abstract}

\section{Keywords}

new products:cross-functional performance metrics, marketing-operations coordination, competitive diffusion dynamics, cost of delay

\section{Disciplines}

Other Business | Other Economics | Sales and Merchandising

\section{Comments}

At the time of publication, author Sergei Savin was affiliated with the Columbia University, New York. Currently (July 2016), he is a faculty member in the Operation, Information and Decision amking Department of the Wharton School at the University of Pennsylvania. 


\title{
Modeling Trade-offs in New Product Development: Competitive Demand Dynamics and the Optimal Time of Product Launch
}

\author{
Sergei Savin \\ Columbia Business School
}

Christian Terwiesch

The Wharton School

March 3, 2003

\begin{abstract}
We present a model describing the demand dynamics of two new products competing for a limited target market. The demand trajectories of the two products are driven by a market saturation effect and an imitation effect reflecting the product experience of previous adopters. In this general setting, we provide analytical results for the sales trajectories and lifecycle sales of the competing products. We use these results to study the impact of launch time delay (acceleration) on overall lifecycle sales. Our analysis support trade-off rules that can be used in the tactical decision-making of a product development team. Taking the perspective of a team developing one of the competing products, we find that the profit-maximizing launch time response exhibits a counter-intuitive behavior, which results from nonconcavities in the underlying objective function. In particular, we show that a firm facing a launch time delay from a competing product might benefit from moving its own product launch forward in time, opposed to using the softened competitive situation to further improve its cost position. We identify conditions under which such non-concavities arise and a marginal cost-benefit analysis leads to sub-optimal launch time decisions. Finally, we analyze the Nash equilibrium in launch time decisions of the two competing brands.

KEYWORDS: New Product Development, Marketing-Operations Coordination, Cross-functional Performance Metrics, Cost of Delay, Competitve New Product Demand Dynamics
\end{abstract}




\section{Introduction}

A common problem in product development is the trade-off between the four performance metrics: product development lead-time, product unit cost, technical product performance, and overall development cost (Smith and Reinertsen 1991, Figure 1a). To find a balance between these conflicting objectives in their day-to-day decision-making, development teams typically rely on simple trade-off rules. Such trade-off rules attempt to 'dollarize' (i.e. assign a financial value to) changes along any of these metrics and thereby to create a common ground for comparison across organizational functions (Ulrich and Eppinger 1999, Figure 1b). However, such a 'dollarization' requires that the development team is able to compute the impact of a change in one of the four performance metrics on the new product's profitability.

While understanding the financial impact of changes in development cost and product unit cost is relatively simple, understanding the financial impact of a change in launch time is rather difficult: what is the cost of a one day launch delay, e.g. in the automotive industry? One million dollars? Maybe two? How can we adequately capture the long-term impact on market share resulting from the launch delay? Clearly, whichever answer we choose will have a substantial impact on the quality of the development team's decision making.

Yet, despite their pivotal role in guiding decision making and cross functional coordination in the development of a new product, the formation of these trade-off rules is commonly done in an ad-hoc fashion. One major shortcoming of the current decision making process is its simplistic treatment of demand dynamics over the product lifecycle. Standard models either assume that the lifecycle demand of the new product is exogenous and therefore not affected by a delayed launch (the sales curve is just shifted into the future) or that there is a pre-determined market window, after which sales are reduced to zero (Ulrich and Eppinger 1999). Factors that have been identified as critical success drivers for a new product, such as time-to-market relative to competition (Porter 1985, Kalish and Lilien 1986), or product diffusion (Bass 1969, Krishnan et al. 2000) are, at best, only included qualitatively (Ulrich and Eppinger 1999).

The first objective of the present manuscript is to overcome this shortcoming by developing quantitative trade-off rules with respect to product development lead-time. In contrast to the simplistic treatment of demand dynamics prevalent in existing models, the trade-off rules we derive are grounded on a detailed analysis of competition and product diffusion, and thereby allow for an endogenous analysis of the lifecycle demand with respect 
to changes in development lead time. Our second objective is to demonstrate how such trade-off rules can be used for coordination between conflicting objectives of marketing and operations.

Our work is contributing to three literature streams discussed in Section 2: product development decisions, normative models of competitive product diffusion, and marketingoperations coordination. Specifically, we provide the following novel results. First, we present closed form expressions for the diffusion of two competing products within the same category modeling market saturation and word-of-mouth effects. Specifically, we derive closed-form expressions for the product lifecycle sales as functions of the time gap between the launch of the two brands (Theorem 1). We also investigate how lifecycle sales are influenced by the values of diffusion parameters at the brand as well as the category level (Theorem 2). We extend previous models of competitive product diffusion by explicitly modeling the interactions (word-of-mouth) between potential adopters of one brand with previous adopters from the same brand as well as with previous adopters from a competing brand. While this effect has been observed empirically (Mahajan et al. 1993), previous research has not formally modeled demand dynamics in the presence of such interactions (See Table 1 for an overview of related research in competitive new product diffusion).

Second, we analyze the coordination between the marketing and the operations efforts of a team developing one of the competing products. In particular, we model the team's decision of finding 'the optimal' time of product launch. From a marketing perspective, the team would like to launch the product sooner, as this would lead to higher unit sales over the lifecycle. From the operations perspective, the team would like to spend additional time on the detailed engineering of the product and the corresponding production process, as this would lead to lower unit costs. We show how a project manager can resolve this tension by deriving (dollarizing) how a change in launch time impacts the profitability of the new product. We derive conditions under which the immediate launch of a new product is optimal. We also demonstrate that there are situations where making the launch time decisions on a marginal profit basis can be misleading: we show that it might be optimal to delay market launch despite a negative marginal value of a longer development time (Theorem 3).

Third, we analyze the existence and the nature of the Nash equilibrium with respect to the market entry times of the two competing products. For the case of completely symmetric products, we derive sufficient conditions for the existence of a Nash equilibrium for which both competitors launch immediately. In addition, we identify the range of cost 
parameters for which the pure strategy equilibrium does not exist. While the existence of a pure Nash equilibrium for arbitrary set of problem parameters is hard to establish analytically, we derive a set of conditions that characterize the nature of the equilibrium for asymmetric players.

The remainder of this manuscript is organized as follows. After reviewing the related literature (Section 2), we present a model of competitive diffusion and formulate the optimization problem for the time-to-market for one of the competitors given the estimated entry time for the other competitor (Section 3). In Section 4 we present closed-form expressions for the sales trajectories and lifecycle sales of the two competing products. Section 5 uses these results to balance the marketing and operations metrics in deciding about the launch time of a new product, followed by our equilibrium analysis presented in Section 6. Section 7 demonstrates the practical implications of our work and Section 8 provides concluding remarks.

\section{Related Literature}

Ulrich and Eppinger (1999) recommend a four-step procedure towards evaluating, among others, the trade-off between development lead-time and cost: (1) Build a base case financial model (including a spreadsheet and a representation of lifecycle demand), (2) Perform sensitivity analysis to understand the key assumptions of the model, (3) Use sensitivity analysis to understand the trade-offs (including the trade-off between cost and time), and (4) Consider the influence of qualitative factors, including competition and other market characteristics.

The strength of this approach is its simplicity and the little effort required for implementation. However, the approach following steps (1)-(3) is biased towards the easily measurable costs, including idle production plants, the cost of capital, and the expenses related to additional development time, while ignoring the 'hidden cost' associated with the negative impact on revenues that results from a delayed launch.

A good example of this approach can be found in Clark (1989), who reports: 'Research indicates that each day of delay in market introduction costs an automobile firm over $\$ 1$ million in lost profits, not including the impact of lost market share.' While such numbers are certainly effective in directing senior management's attention to even minor launch delays, they are of little value when guiding development teams in their operational decisions. Whether or not a development team in the automobile industry around 1989 would be well advised in spending $\$ 1.5$ million to avoid a one day launch delay depends 
on how large the market share loss really turns out to be. Thus, a more detailed model is needed, that goes beyond treating the market side as a qualitative factor or a residual.

Several authors provide qualitative guidelines on how revenues are affected by a slow (delayed) versus a fast (accelerated) launch, four of which are displayed in Figure 2. Urban and Hauser (1993) argue that reducing lead-time will increase lifecycle sales, however, with diminishing returns. It is also suggested that an accelerated launch will increase cost as well as risk. Given that additional time in development reduces cost at a diminishing rate, the graph suggests the existence of an optimal launch time. While Urban and Hauser do emphasize the importance of competition, neither sequence of entry nor the duration of the first mover monopoly is visible in their graph.

Rosenthal (1992) takes a slightly different approach. Based on a forthcoming competitor's product introduction, a late launch will give the product a shorter growth period, and thereby smaller peak sales. Moving from actual (late) introduction to the earlier, planned introduction seems to indicate increasing returns (peak moves up, so does duration of the monopoly period). The framework explicitly includes competition - assumed to begin at the point in time when sales start to fall - and takes the perspective of the first mover.

Kalyanaram and Krishnan (1997) suggest a convex-concave relationship between leadtime and sales. The convex part of their graph, the authors argue, results from the product diffusion in its monopoly phase. The switching point (from convex to concave) indicates the beginning of the competitive phase. Similar to Rosenthal's model, the authors include competition and take the perspective of the first mover.

Finally, Wheelwright and Clark (1992) argue that there are steep gains associated with shortened development lead-times, especially for companies who are 'head to head' with their competition. Getting too far ahead does not yield the desired increase in profits, and can even result in profit loss.

Taken together, all four graphs in Figure 2 emphasize the impact of changes in product development lead-time on a product's lifecycle sales and profits. However, by contrasting the four graphs, we can make a couple of interesting observations. First, all four curves are purely qualitative and support, at best, step (4) in the Ulrich and Eppinger procedure. Since none of the curves is described in a functional form, they are impossible to use for quantitative decision-making. Second, none of the four graphs is derived formally from a transparent set of assumptions, making it hard for a project manager to judge if the corresponding model fits her current situation. In particular, the effects of competition and diffusion (the main differentiators from spreadsheet based models) are included in a rather informal manner. Finally, a comparison of the four graphs in Figure 2 is difficult, 
since the graphs differ with respect to both the horizontal axis and the graph plotted along the vertical axis. We will revisit Figure 2 in Section 7, where we display our analytical results in the formats used by Rosenthal as well as by Urban and Hauser.

\section{Detailed Models of Market Demand}

As a first step towards developing a model of competition and diffusion, we turn to the marketing literature, which provides a rich stream of research related to product introduction and demand dynamics, specifically in the area of new product diffusion models $\left(\right.$ Bass 1969) ${ }^{1}$. The Bass model leads to the S-shaped product diffusion that has commonly been reported for new product categories: sales for a new product are initially low, as there exists limited word-of-mouth for it, and customers only adopt the product in response to external influences (captured by the coefficient of innovation). With more customers adopting the product, the word-of-mouth effect for the new product becomes stronger and the sales rate increases (captured by the coefficient of imitation). Finally, the sales rate starts to decrease, reflecting the overall market saturation.

A model of category level diffusion is important when analyzing the demand dynamics of two competing products within a category. For example, Krishnan et al. (2000) discuss how Chrysler introduced the Caravan and the Voyager (brands) back in 1984 and thereby acted as a pioneer in the minivan (category) market. When a few years later Ford introduced the Aerostar, Ford was able to benefit from the category awareness for minivans and captured a sizable portion of the market. Thus, although the first product to market obtains $100 \%$ of the category sales up to the arrival of the second product, initial sales can be low given the limited awareness for the new category. We will label this effect as the category-awareness effect.

Whereas the original models of new product diffusion were applied at the product category level, the last fifteen years have witnessed the evolution of a significant body of research on diffusion models incorporating the effects of brand competition within a category (see Chatterjee et al. 1998 for an overview of competitive diffusion models). There are two effects of brand-level diffusion that are important to consider when modeling the impact of launch time on demand dynamics, brand-level word-of-mouth and cross-brand word-of-mouth.

In addition to the word-of-mouth effect at the category level, customers also exchange information at the brand level. Consider a customer deciding which brand within the mobile phone category to adopt. A customer who has interacted with a prior adopter of

\footnotetext{
${ }^{1}$ We will review Bass's mathematical model further below. For an overview of the Bass model as well as its numerous extensions, we refer the reader to Mahajan et al (1990).
} 
Brand A will not only be more likely to also buy any mobile phone (category-awareness effect) but will be more (or less) likely to also adopt Brand A. To capture this effect, Krishnan et al. (2000) extend the traditional Bass model to the brand level and assume that each brand has its own coefficient of innovation as well as coefficient of imitation. The coefficient of imitation in the model presented by Krishnan et al. captures the effect that prior adopters of the category have on the future adopters of a brand as a '...collective force of all previous adopters that act on each brand's future adoption (Krishnan et al. 2000 , p. 271)'. While lumping the effects of brand-level word-of-mouth together into a collective force makes the resulting diffusion equations more elegant, it does not separate between the word-of-mouth for brand A coming from customers who have adopted brand A vs. customers who have adopted brand B.

However, such a separation can be important, especially when studying the sales loss of the incumbent as a result of a new market entrant. This observation is in line with the empirical work by Mahajan et al. (1993), who specify a model of competition and diffusion, which they use to assess the impact of competitive entry on the incumbent's sales. The model specification for the empirical analysis by Mahajan et al. (1993) not only includes the word-of-mouth between potential adopters of one brand with previous adopters from the entire category but explicitly models the 'cross brand word-of-mouth' of both brands separately. The model is used to study the competition between Polaroid and Kodak and to evaluate Polaroid's sales loss as a result of Kodak's patent infringement. As we share Mahajan et al.'s interest in understanding the impact of competitive entry on the incumbent's sales dynamics, we incorporate their concept of 'cross brand word-of-mouth' into our analysis.

Despite the successful use of competitive Bass-type diffusion models empirically, only three prior articles have presented normative models related to the timing of entry within a competitive diffusion context (Chatterjee et al. 1998). The three studies are compared in Table 1, together with the two most relevant empirical studies as well as the present manuscript. Eliashberg and Jeuland (1986) consider a pioneering brand and a competing brand introduced at a later point. Their study takes the launch times as exogenous and focus on pricing decisions. The underlying demand model is characterized by a saturation effect, but does not include a word-of-mouth effect. Similarly, Fershtman, Mahajan and Muller (1990) focus on pricing decisions, but also include an advertising decision. Unlike Eliashberg and Jeuland (1986), their demand model is characterized by a word-of-mouth effect, but does not include a saturation effect. The only competitive diffusion model with saturation and word-of-mouth effect is provided by Kalish, Mahajan, and Muller (1995). 
Yet, given that the authors analyze the timing of entry in different global markets, they do not provide analytical results on how a change in launch time influences the lifecycle sales and profitability of the product.

\section{Marketing-Operations Coordination}

From the operations perspective, an additional day in development provides the opportunity to spend more time on fine-tuning the product and its corresponding manufacturing process. Bhattacharya et al. (1998) distinguish between extra time that is used towards improving the product performance of the new product (concept definition) and time that is used to reduce product unit cost for a given concept (set of features). In our analysis, we assume that the product concept is frozen and that all additional time will be spent towards reducing the product's unit cost. We thereby model the trade-off between launch time and one out of the three performance dimensions in Figure 1. Extending this analysis to either include a third dimension, or to include a different second dimension (product performance or development cost) is interesting, yet beyond the scope of this paper.

From the marketing perspective, an additional day in development will typically not be welcome. The pool of potential customers is shrinking because of current or future competition (market saturation effect). Moreover, the product category diffusion will commence (category level word-of-mouth effect) and the competing product will have an opportunity to receive more brand-level word of mouth (brand level word-of-mouth effect). Given these conflicting objectives, a decision maker needs to compare foregone profit margins resulting from a delayed launch with reduced unit costs over the entire product lifecycle. If engineers improve unit cost corresponding to $\$ 500 \mathrm{~K}$ cost savings for each extra day while the market is shrinking with a financial impact of $\$ 1$ million each day, it is probably time to launch. Thus, a 'dollarization' of delay creates a truly crossfunctional performance metric, leading to improved coordination between marketing and operations.

However, there are situations where attempts of coordinating marketing and operations based on marginal analysis can lead to overall Pareto-inefficiencies. De Groote (1994) presents a model in which the operations function chooses an optimal batch size and a production technology, which is driving the set-up costs per batch, while marketing chooses the degree of product variety. In this set-up, each of the two functions fully takes into account its impact on the other function, thus coordination inefficiencies do not arise from ignorance. However, as their choice is based on a marginal analysis (What is the degree of variety for the current production technology? What is the optimal production technology for the current degree of variety?), the firm as a whole may forego a global 
performance maximum (higher variety with more flexible production technology). Other studies pointing at a need for a more integrated decision making include Schmidt and Porteus (2000) and Cohen et al. (2000).

\section{Model Formulation}

Using a model of competitive product diffusion similar to the one underlying the empirical studies by Krishnan et al. (2000), we consider the competitive diffusion of two products (1 and 2). At $t=0$, the manager responsible for the launch of product 1 , is faced with the decision of when to launch her product, provided that the launch time of the competing product $2, T_{2}$, is known. $T_{2}$ may be positive or negative, reflecting the fact that product 2 may yet to be launched or may be launched already. Define the beginning of the competitive diffusion as $t_{\mathrm{c}}=\max \left(T_{2}, T_{1}\right)$.

We assume that the two competing products jointly constitute a product category and compete for the same population of potential adopters of size $m$. We assume the population of potential adopters to be independent of the number of products currently on the market, which allows us to isolate the saturation effect discussed above. Thus, when the second product enters, the rate of diffusion will change, but not the market potential. Our model would have to be extended to capture the case that a new product entering the market not only alters the diffusion rate, but also increases the population of potential adopters (see Krishnan et al. 2000 for an excellent discussion of these two effects) ${ }^{2}$.

The competitive new product diffusion process is specified as follows. Sales of product $i$ reflect product adoption by three different groups of adopters: 'innovators', who adopt product $i$ based on an external influence, 'imitators', who adopt due to networking interaction (internal influence) with those who already adopted the same product, and 'cross-imitators', who adopt product $i$ after interacting with those who already adopted product $j$. This adoption process is illustrated in Figure 3 . For $t \geq t_{\mathrm{c}}$, denote the cumulative sales function of product $i$ by $D_{i}(t), i=1,2$. The sales rate of product $i$ depends on the cumulative sales of both products by time $t$ :

$$
\begin{aligned}
& \frac{d D_{1}}{d t}=\left(p_{1}+\frac{1}{m}\left(q_{11} D_{1}(t)+q_{12} D_{2}(t)\right)\right)\left(m-D_{1}(t)-D_{2}(t)\right), \\
& \frac{d D_{2}}{d t}=\left(p_{2}+\frac{1}{m}\left(q_{21} D_{1}(t)+q_{22} D_{2}(t)\right)\right)\left(m-D_{1}(t)-D_{2}(t)\right) .
\end{aligned}
$$

\footnotetext{
${ }^{2}$ Closed form solutions for our model can still be obtained for that generalized case as long as the population of potential adopters is a constant $m$ for $t<t_{\mathrm{c}}$ and a (different) constant $M$ for $t \geq t_{\mathrm{c}}$. Note that this formulation does include the Krishnan et al. (2000) model.
} 
Diffusion equations (1) represent an extension of the diffusion dynamics in Bass (1969) and reflect the interaction between the individual diffusions for products 1 and 2 through both the market saturation effect $m-D_{1}(t)-D_{2}(t)$, as well as through the word-ofmouth effect. The word-of-mouth effect occurs both at the category level as well as at the brand level. Specifically, $q_{i j}$ defines the probability that a customer adopts product $i$ after having interacted with a previous adopter of product $j$. Mahajan et al. (1993) label this coefficient - for $i \neq j$-as the brand competition effect ${ }^{3}$.

We assume that the sales of the entire product category $D(t)=D_{1}(t)+D_{2}(t)$ follow the classical Bass pattern. We observe that (3) describes a Bass diffusion only if

$$
q_{11}+q_{21}=q_{12}+q_{22}=q
$$

where $q$ captures the category level word-of-mouth effect. The assumption of brand level diffusions jointly constituting a category level diffusion follows the argument by Krishnan et al. (2000) who convincingly argue: 'The property that the proposed brand-level model sums up to the Bass category-level model may look restrictive, but it gives indirect face validity to the proposed model because the Bass (1969) model has a strong behavioral basis and has found excellent empirical support over a wide range of products'.

Adding the two equations in the (1), we obtain:

$$
\frac{d D}{d t}=\left(p+\frac{1}{m}\left(\left(q_{11}+q_{21}\right) D_{1}(t)+\left(q_{12}+q_{22}\right) D_{2}(t)\right)\right)(m-D(t)) .
$$

where $p=p_{1}+p_{2}$.

Define $\beta=\frac{p_{1}}{p}$ as the probability that an 'innovative' customer will select product 1 , given that she decided to adopt one of the two products. The coefficient of external influence, $p$, in a diffusion captures all time-invariant effects on the adoption decision of the customer. This includes the absolute attractiveness of the product, the awareness of the product created independent of market share (e.g. media advertising), or the price position of the new product. Consequently $\beta$ provides a measure to what extent the external influence of the diffusion favors brand 1 versus brand 2 . If $\beta=0.5$, both products experience the same external influence.

Further, define $\alpha_{1}=\frac{q_{11}}{q}$ and $\alpha_{2}=\frac{q_{22}}{q}$. The $\alpha_{i}$ describe the imitation process for the respective product brands and can be interpreted as measures of relative attractiveness for potential adopters. We can then express (1) as

$$
\frac{d D_{1}}{d t}=\left(p \beta+\frac{q}{m}\left(\alpha_{1} D_{1}(t)+\left(1-\alpha_{2}\right) D_{2}(t)\right)\right)\left(m-D_{1}(t)-D_{2}(t)\right),
$$

\footnotetext{
${ }^{3} \mathrm{~A}$ similar functional form is also used in models of multi-product diffusion (see Peterson and Mahajan 1978, Bayus et al. 2000)
} 


$$
\frac{d D_{2}}{d t}=\left(p(1-\beta)+\frac{q}{m}\left(\left(1-\alpha_{1}\right) D_{1}(t)+\alpha_{2} D_{2}(t)\right)\right)\left(m-D_{1}(t)-D_{2}(t)\right) .
$$

We note that the general set of diffusion equations (4) includes two important competitive diffusion regimes as particular cases. On the one hand, when $\alpha_{1}, \alpha_{2} \rightarrow 0.5$, the competitive diffusion proceeds mainly at the level of the entire category - in this case, the 'imitative' consumers look at each of the competitors as members of a particular category, rather than individual products. On the other hand, when $\alpha_{1}, \alpha_{2} \rightarrow 1$, the diffusion shifts to the level of the individual brands.

Similar to prior studies in this field (Bayus et al. (1997), Krishnan et al. (2000), Mahajan et al. (1993)), our analysis treats the diffusion coefficients $p_{i}$ and $q_{i j}$, and thereby $\alpha_{1}, \alpha_{2}$, and $\beta$, as exogenously given. Given the extensive body of research on diffusion models in various industries (e.g. Van den Bulte 2000), it is possible to obtain good predictions for the corresponding diffusion parameters ${ }^{4}$. Since the values of the diffusion parameters can be influenced by marketing mix variables, such as advertising and pricing (see Bass et al. 1994, Bass et al. 2000), using past diffusion parameters to describe the projected sales of a new product implicitly assumes that the marketing decisions involved in managing the sales processes of new products will be predictable and similar to those used in the past. This assumption allows us to isolate the effects of launch time delays on the life-cycle sales of a new product in a competitive environment ${ }^{5}$.

Product 1 may be introduced at any time $T_{1} \geq 0$. Let $T=T_{2}-T_{1}$ denote the launch time gap between the two products: $T>0$ indicates that product 1 is introduced ahead of product 2 , while $T<0$ corresponds to the case when product 2 is introduced first. Note that, since $T_{1} \geq 0, T \leq T_{2}$. Since by $t=t_{\mathrm{c}} \geq 0$ one of the products enjoyed a monopoly setting, the initial conditions for (1) are

$$
\left(D_{1}\left(t=t_{\mathrm{c}}\right), D_{2}\left(t=t_{\mathrm{c}}\right)\right)=\left(\bar{D}_{1}(T), \bar{D}_{2}(T)\right)
$$

where

$$
\begin{gathered}
\bar{D}_{1}(T)= \begin{cases}0, & T \leq 0, \\
m\left(1-\frac{p \beta+q \alpha_{1}}{q \alpha_{1}+p \beta \exp \left(\left(p \beta+q \alpha_{1}\right) T\right)}\right), & T>0,\end{cases} \\
\bar{D}_{2}(T)= \begin{cases}m\left(1-\frac{p(1-\beta)+q \alpha_{2}}{q \alpha_{2}+p(1-\beta) \exp \left(\left(p(1-\beta)+q \alpha_{2}\right)|T|\right)}\right), & T \leq 0, \\
0, & T>0,\end{cases}
\end{gathered}
$$

\footnotetext{
${ }^{4}$ If the parameters are not known at the beginning of the diffusion, one would have to rely on an updating mechanism to improve the estimates dynamically (see e.g. Xie et al. 1997).

${ }^{5}$ See Bass et al. (1994) for an excellent overview on how marketing mix decisions relate to product diffusion.
} 
are the cumulative sales of product $i$ prior to competition (Bass 1969). If both sales processes start simultaneously, $T=0$ and $\left(D_{1}\left(t=t_{\mathrm{c}}\right), D_{2}\left(t=t_{\mathrm{c}}\right)\right)=(0,0)$. This set-up assumes that the product first to market will follow a Bass diffusion, which means that consumers are not anticipating - and potentially delaying - their adoption decision because of the future launch of the product second to market ${ }^{6}$.

Our analysis will take the position of a firm launching product 1 . We are interested in obtaining expressions for the lifecycle sales $M_{1}(T)=D_{1}(+\infty)$, and, in particular, in quantifying the impact of a delayed launch on the lifecycle sales $d M_{1}(T) / d T$. As we do not model changes in prices and production costs over the course of the lifecycle, the overall lifecycle sales also provides a measure of profits derived from the new product.

While an extended development time will have negative consequences through reduced lifecycle sales, such an extension might be warranted if it is connected with operational improvements. We assume that there exists a constant return to cost reduction effort, thus unit cost for product 1 can be written as:

$$
c_{1}\left(T_{1}\right)=\delta_{0}+\delta_{1} e^{-\gamma T_{1}}
$$

where $\delta_{0}, \delta_{1}$, and $\gamma$ are all non-negative. In (8), $\delta_{0}+\delta_{1}$ denotes the unit cost if product 1 is launched immediately, while $\delta_{0}$ corresponds to the minimum possible unit cost. $\gamma$ denotes the rate with which the development team can reduce cost. As reflected in (8), we assume that the per unit costs are driven largely by the product design, opposed to experience effects (learning curves) in the manufacturing facility. This assumption is consistent with Eliashberg and Jeuland (1986) and Kalish et al. (1995). In contrast, Fershtman et al. (1990) allow for volume learning, i.e. unit cost falling with cumulative output. None of the previous studies considers cost reduction opportunities resulting from a delayed launch.

Denoting by $\pi$ the average (over the life-cycle period) selling price for product 1 , we can express the profit margin of product 1 given launch time $T_{1}$ :

$$
\sigma\left(T_{1}\right)=\pi-\delta_{0}-\delta_{1} e^{-\gamma T_{1}}=\left(\pi-\delta_{0}\right)\left(1-c e^{-\gamma T_{1}}\right)
$$

where $c=\frac{\delta_{1}}{\pi-\delta_{0}}$. Here we assume that, irrespective of chosen launch time, the profit margin remains non-negative, so that $\pi>\delta_{0}$ and $0 \leq c \leq 1$. Combining our demand model given by (4) and (5) with the model of cost reduction in (8), we can state the optimization problem for the firm launching product 1 as:

$$
P=\max _{T \leq T_{2}}\left(M_{1}(T)\left(1-c e^{\gamma\left(T-T_{2}\right)}\right)\right)
$$

\footnotetext{
${ }^{6}$ See Eliashberg et al. (2002) for how the announcement of a new product impacts the diffusion pattern.
} 
The optimization model (10) focuses on the optimal response of a team developing a new product to the market entry of a competitor, which has happened in the past $\left(T_{2}<0\right)$ or is expected to happen in the future $\left(T_{2}>0\right)$. The central part of the analysis of such optimal response relies on the properties of the lifecycle sales function $M_{1}(T)$, which we analyze in the following section.

\section{The Impact of Launch Time on Lifecycle Sales}

The competitive diffusion described by (1) has one important property (Lemma 1): for every value of the launch gap $T \neq 0$, sales of both products can be shown to be equivalent to those for some synchronous $(T=0)$ diffusion. Define the remaining market potential $\bar{m}(T)$ for a given launch time $T$ as:

$$
\bar{m}(T)=m-\sum_{i=1}^{2} \bar{D}_{i}(T) .
$$

Moreover, define the 'launch time adjusted' coefficients of innovation and imitation as:

$$
\begin{gathered}
\bar{p}_{i}(T)=p_{i}+\sum_{j=1}^{2} \frac{q_{i j}}{m} \bar{D}_{j}(T), \forall i=1,2, \\
\bar{q}_{i j}(T)=\frac{q_{i j}}{m} \bar{m}(T), i, j=1,2
\end{gathered}
$$

The sales of the competing products can be expressed as follows:

Lemma 1: Denote $\mathbf{p}=\left(p_{1}, p_{2}\right)$ and $\mathbf{q}=\left(q_{11}, q_{12}, q_{21}, q_{22}\right)$. Also, for $T \neq 0$, denote by $D_{i}(t, T, \mathbf{p}, \mathbf{q}, m), i=1,2$ the solution to (1) subject to initial conditions $(5), t \geq t_{\mathrm{c}}$. Then,

$$
D_{i}(t, T, \mathbf{p}, \mathbf{q}, m)=D_{i}\left(t-t_{\mathrm{c}}, 0, \overline{\mathbf{p}}(T), \overline{\mathbf{q}}(T), \bar{m}(T)\right)+\bar{D}_{i}(T), i=1,2, t \geq t_{\mathrm{c}}
$$

Lemma 1 stipulates that the diffusion patterns for two products entering the market at different points in time can be reduced to diffusion patterns with a simultaneous market entry. The transformation (14) is intuitive. The market size for the phase with both products on the market needs to be reduced to account for the sales that the pioneering product could realize before the beginning of competition. Moreover, the customers who have adopted the pioneering product will create and additional external influence on consumers for the pioneering product, which increases the corresponding coefficient of innovation.

Based on the definitions (11)-(13), we can make two interesting observations. First, unlike Kalish et al. (1995), our demand dynamics of the overall category diffusion are 
still consistent with the traditional Bass dynamics as outlined in Bass (1969). Second, while the transformations (11)-(13) change both the market size and the imitation parameters, the imitation and cross-imitation components of the diffusion dynamics $\frac{\bar{q}_{i j}(T)}{\bar{m}(T)}$ remain unchanged.

Using the 'launch gap' transformations introduced above, we can establish the sales trajectories of the competing products as well as their lifecycle sales for any combination of the launch gap and the diffusion parameters. Towards this goal, define $\bar{D}(T)=\bar{D}_{1}(T)+$ $\bar{D}_{2}(T)$ using the transformation defined by (6) and (7).

\section{Theorem 1}

(a) Let $D(t)=m\left(1-\frac{q+p}{q+p \exp ((p+q) t)}\right)$ denote the cumulative sales for the entire product category at time $t$. Then, if there is a market entry gap between two products $T=T_{2}-T_{1}$, the cumulative sales of competing brands are given by

$$
\begin{aligned}
& D_{1}(\bar{t})=\left\{\begin{array}{l}
\left(\bar{D}_{1}(T)-\frac{\left(1-\alpha_{2}\right) \bar{D}(T)}{2-\alpha_{1}-\alpha_{2}}+\frac{p m}{q\left(\alpha_{1}+\alpha_{2}-1\right)}\left(\beta-\frac{\left(1-\alpha_{2}\right)}{\left(2-\alpha_{1}-\alpha_{2}\right)}\right)\right)\left(\frac{1+\frac{q D(\bar{t})}{p m}}{1+\frac{q \bar{D}(T)}{p m}}\right)^{\alpha_{1}+\alpha_{2}-1} \\
+\frac{\left(1-\alpha_{2}\right)}{\left(2-\alpha_{1}-\alpha_{2}\right)} D(\bar{t})-\frac{p m}{q\left(\alpha_{1}+\alpha_{2}-1\right)}\left(\beta-\frac{\left(1-\alpha_{2}\right)}{\left(2-\alpha_{1}-\alpha_{2}\right)}\right) \\
\alpha_{1}+\alpha_{2} \neq 1, \alpha_{1}+\alpha_{2}<2 \\
\bar{D}_{1}(T)+\alpha_{1}(D(\bar{t})-\bar{D}(T))+\frac{p m\left(\beta-\alpha_{1}\right)}{q} \log \left(\frac{1+\frac{q D(\bar{t})}{p m}}{1+\frac{q \bar{D}(T)}{p m}}\right) \\
\alpha_{1}+\alpha_{2}=1 \\
\frac{\left(p \beta+\frac{q}{m} \bar{D}_{1}(T)\right) D(\bar{t})+p\left(\bar{D}_{1}(T)-\beta \bar{D}(T)\right)}{p+\frac{q}{m} \bar{D}(T)}, \alpha_{1}+\alpha_{2}=2
\end{array}\right. \\
& D_{2}(\bar{t})=D(\bar{t})-D_{1}(\bar{t}),
\end{aligned}
$$

(b) For any $T$, the lifecycle sales for product 1, $M_{1}(T)$, are given by:

$$
M_{1}(T)=\left\{\begin{array}{l}
\left(\bar{D}_{1}(T)-\frac{\left(1-\alpha_{2}\right) \bar{D}(T)}{2-\alpha_{1}-\alpha_{2}}+\frac{p m}{q\left(\alpha_{1}+\alpha_{2}-1\right)}\left(\beta-\frac{\left(1-\alpha_{2}\right)}{\left(2-\alpha_{1}-\alpha_{2}\right)}\right)\right)\left(\frac{1+\frac{q}{p}}{1+\frac{q \bar{D}(T)}{p m}}\right)^{\alpha_{1}+\alpha_{2}-1} \\
+\frac{\left(1-\alpha_{2}\right)}{\left(2-\alpha_{1}-\alpha_{2}\right)} m-\frac{p m}{q\left(\alpha_{1}+\alpha_{2}-1\right)}\left(\beta-\frac{\left(1-\alpha_{2}\right)}{\left(2-\alpha_{1}-\alpha_{2}\right)}\right) \\
\alpha_{1}+\alpha_{2} \neq 1, \alpha_{1}+\alpha_{2}<2 \\
\bar{D}_{1}(T)+\alpha_{1}(m-\bar{D}(T))+\frac{p m\left(\beta-\alpha_{1}\right)}{q} \log \left(\frac{1+\frac{q}{p}}{1+\frac{q \bar{D}(T)}{p m}}\right) \\
\alpha_{1}+\alpha_{2}=1 \\
\frac{\left(p \beta+\frac{q}{m} \bar{D}_{1}(T)\right) m+p\left(\bar{D}_{1}(T)-\beta \bar{D}(T)\right)}{p+\frac{q}{m} \bar{D}(T)}, \alpha_{1}+\alpha_{2}=2
\end{array}\right.
$$

The above result specifies the cumulative sales of each of the competing products at any point in time as well as the values of the lifecycle sales acquired at the end of the competitive diffusion period. We observe that while the sales dynamics of the entire category follows the Bass pattern, the individual product sales can be very different from 
it. Figures 4 and 5 show how the demand rate for competitor 1 is influenced by the market entry time of the competitor 2 for the cases when the competitive diffusion proceeds on the level of the entire product category $\left(\alpha_{1}=\alpha_{2}=0.5\right.$, Figure 4) or, almost entirely, on the brand level $\left(\alpha_{1}=\alpha_{2}=0.9\right.$, Figure 5).

Figure 4 indicates that while the position of the demand peak for competitor 1 is pushed into the future as the entrance of the competitor 2 is delayed, the height of this demand peak is not influenced by the competitor's entry time unless the competitor is starting very far ahead. This last feature is a direct consequence of the 'category' nature of the competitive diffusion process. Note that the competitor's entry creates a kink in the sales curve most visible for the curves corresponding to $T=10$ and $T=20$. Figure 5 demonstrates that for the 'brand level' diffusion both the position of the demand peak and its height are, as expected, monotone functions of competitor's entry time.

The closed-form expressions (16) for lifecycle sales are of fundamental importance for our analysis of the impact of launch time on lifecycle sales. This impact can be quantified by evaluating monotonicity and curvature of lifecycle sales as a function of launch time $T$. Theorem 2 captures these properties.

\section{Theorem 2}

(a) Lifecycle sales for product 1, $M_{1}(T)$, increases with relative product attractiveness, $\alpha_{1}$, and the value of external influence, $\beta . M_{1}(T)$ decreases with launch delay $T$ and the relative attractiveness of the competing product, $\alpha_{2}$.

(b) For any $0<\alpha_{1}, \beta<1$, there exists a launch delay value $\bar{T}_{\max }$ such that $M_{1}(T)$ is a concave function of $T$ for $T \geq \bar{T}_{\max }$. In addition, there exists a launch delay value $\bar{T}_{\min }<\bar{T}_{\max }$ such that $M_{1}(T)$ is a convex (concave) function of $T$ for $0 \leq T \leq \bar{T}_{\min }$ provided that $\frac{p}{q}<(>) \frac{\alpha_{1}}{\beta}+\frac{1-\alpha_{1}}{1-\beta}-\alpha_{1}-\alpha_{2}$.

As expected, lifecycle sales of product 1 decrease with its launch delay. A similar effect is observed when the relative attractiveness of the product, as measured by the ratio of its $\alpha$ coefficient to that one of the competing product, increases. Increases in the strength of the external influence, $\beta$, will also lead to an increase in product's lifecycle sales.

Part (b) of Theorem 2 states the properties for the lifecycle sales of product 1 for the case when it is introduced ahead of product 2 . The analogous statements for the case when product 2 is introduced first, are easily obtained by interchanging $\alpha_{1}$ and $\alpha_{2}$ as well as $\beta$ and $1-\beta$ in the above expressions and using the 'conservation' condition $M_{1}(T)+M_{2}(T)=m$.

We observe that lifecycle sales of product 1 as a function of its launch delay can exhibit 
a 'non-concave' behavior. In other words, the marginal impact of a launch delay does not have to be monotone. Such non-concavities are of importance for the structure of the optimization problem (10) and will strongly impact the marketing-operations coordination analyzed in the following section. This may happen, in particular, in cases when competing products with similar relative attractiveness levels are subject to very dissimilar external influences, or similar external influences exist for products with very different relative attractiveness levels. For example, in the case of $\alpha_{1}=\alpha_{2}=0.8, \beta=0.7$ ('attractive' products, external influence for product 1 heavily dominates that of product 2) the "nonconcavity' occurs for $\frac{p}{q}<0.21$ - a condition which is satisfied for the vast majority of products (for example, the average estimated $\frac{p}{q}$ ratio for the new durable products in the seminal Bass (1969) paper is around 0.05). Similarly, in the case when $\beta=0.5$ and $\alpha_{1}=0.7, \alpha_{2}=0.2$ (equal external influence in the situation when product 1 is relatively more attractive than product 2 ) the 'non-concavity' condition of Theorem $2, \frac{p}{q}<1.1$, is clearly satisfied.

Theorems 1 and 2 enable us to address the questions that we raised in our discussion of Figure 2 by analyzing the impact of different diffusion parameters and entry times on the product's lifecycle sales. The following analysis uses parameter ranges based on the category diffusion as estimated by Bass (1969). Specifically, we assume a coefficient of innovation $p=0.0163221$, a coefficient of imitation of $q=0.325044$, and a market potential of $m=41,298,400$. Moreover, in order to isolate the effect of launch time, we assume that both products are subject to the same degree of external influence $(\beta=0.5)$.

Figure 6 plots the marginal loss in lifecycle sales as a function of the launch delay $T$. Consider first the case where the word-of-mouth predominantly occurs at the brand level ( $\alpha_{1}$ and $\alpha_{2}$ are both fairly large, here chosen as $\alpha_{1}=\alpha_{2}=0.9$ ). At $T=0$, a one unit acceleration in launch time will lead to a 0.08 increase in lifecycle sales. The benefit of an early launch is not only the market-saturation effect, but it is also one of creating a customer base which will lead to an increase in future sales rates as a result of positive word-of-mouth at the brand-level. However, as can be seen by moving from the origin to the left, further acceleration exhibits diminishing returns. This is consistent with the argument by Urban and Hauser (moving from zero to the left). Moreover, as was argued by Kalyanaram and Krishnan, the maximum loss occurs indeed when both competitors are launching head to head $(T=0)$.

If we move the word-of-mouth effect from the brand-level to the category level $\left(\alpha_{1}=\right.$ $\alpha_{2}=0.7$ and $\alpha_{1}=\alpha_{2}=0.3$ ), something interesting happens. The single peak of Figure 6 breaks up into two symmetric peaks, one of each side of the origin $(T=0)$. These peaks 
'drift apart' with a further reduction in $\alpha_{i}$. This reflects the now weaker word-of-mouth effect of the brand. New customers adopt the category, but do so without considering which brand has currently the larger installed base. Thus, customers think of the product they purchase as the category (minivan) opposed to the brand (Caravan, Aero). This substantially weakens the position of the incumbent compared to the case with high $\alpha_{i}$.

Figure 6 shows that - although any acceleration in product launch is beneficial $\left(\frac{d M_{i}}{d T}>0\right)$ - such benefits do not necessarily exhibit diminishing returns. Especially for low values of $\alpha_{i}$, we observe two maxima in Figure 6 , thus $\frac{d^{2} M_{i}}{d^{2} T^{2}}$ can be positive or negative. Hence, in a case where consumers think of a product as a member of a category, opposed to a unique brand, a development team facing head-to-head competition concerning market entry time $(T=0)$ looses fewer units of lifecycle sales per unit launch delay than one would intuitively expect. An application of marginal trade-off rules when coordinating with the operations decision on how much time should be spent on unit cost reduction is thereby likely to lead to a local, sub-optimal maximum.

Figure 7 analyzes the cost of delay for varying levels of word-of-mouth, $q$, for the overall product category. For the same external influence, higher levels of word-of-mouth are associated with a faster product diffusion. As indicated by Figure 7, a one time-unit delay at $T=0$ in the case of $q=0.325$ corresponds to a roughly $3 \%$ loss in lifecycle sales. For $q=0.625$, in contrast, this loss increases to well over $5 \%$ loss in lifecycle sales per unit of delay-time.

Figures 6 and 7 have considered two products of equal desirability $\left(\alpha_{1}=\alpha_{2}\right)$. Figure 8, in contrast, considers the case where product 1 is more desirable than product 2 (in this case $\left.\alpha_{1}=0.8 ; \alpha_{2}=0.6\right)$. We observe that the symmetry from Figures 6 and 7 is replaced by an asymmetric 'double-hump', as described by Wheelwright and Clark. This has the following implications. If product 1 would be able to accelerate its development process, it would introduce a strong product as the first entrant, which would be rewarded with substantial gains in lifecycle sales (up to a $3 \%$ gain in lifecycle sales per unit acceleration). On the other hand, given the attractiveness of product 1, product 1 can afford a delayed entry. Even in presence of a relatively large installed base of product 2, a large number of residual potential adopters would still purchase product 1 ( $q_{12}$ is large). However, for large values of launch delays of product 1, the market saturation effect becomes dominant, and even a superior product is facing limited market potential. The case of different levels of product attractiveness provides an interesting extension opportunity for the model presented above. While in our model, the development team spends additional time on cost reduction effort, one could develop an alternative model in which a team used extra 
development time to increase the product's relative attractiveness.

In summary, our formal model is able to explain some of the apparent inconsistencies that exist in the prior literature as displayed in Figure 2. Previous results were not 'right' or 'wrong', they were simply under-specified, thereby making latent assumptions about the underlying levels of product attractiveness (low vs. high levels of $\alpha_{1}$ and $\alpha_{2}$ ) and diffusion dynamics (word of mouth at the brand vs. at the category level).

\section{Optimal Time-to-Market Responses}

Now, consider a development team which faces the following trade-offs. From the market side, an additional week delaying the product launch would lead to a loss of $\$ 150 \mathrm{~K}$ resulting from foregone revenue margins. At the same time, an additional week delaying the product launch would allow for further effort in DFM activity, leading to a $\$ 100 \mathrm{~K}$ savings in manufacturing costs. Is it time to launch? The costs of delay seem to outweigh the benefits. However, such a marginal analysis can be misleading, as - due to non-concavities in the underlying objective function - multiple local optima can exist. This requires a more detailed analysis of the optimal launch time decision, which is presented in the following Theorem.

Theorem 3 (a) The optimal launch gap $T^{*}$ is a non-decreasing function of $T_{2}$ and $\alpha_{2}$, and a non-increasing function of $c$. Also, there exists $\gamma^{*}$ such that $T^{*}$ is a non-increasing (non-decreasing) function of $\gamma$ for $\gamma<\gamma^{*}\left(\gamma>\gamma^{*}\right)$.

(b) Let $\alpha_{1}+\alpha_{2} \neq 1, \alpha_{1}+\alpha_{2}<2$. For given $p, q, \alpha_{1}, \beta$ and $T_{2}$, define $\widehat{T}=\frac{1}{p \beta+q \alpha_{1}} \log \left(\frac{q \alpha_{1}}{p \beta}\right)$ as the time of the peak demand in product 1 monopoly diffusion and

$$
h^{*}= \begin{cases}\frac{p^{2} \beta(1-\beta)}{(p+q)}, & T_{2}<2 \widehat{T}, \\ \frac{p^{2} \beta(1-\beta)}{(p+q)} \frac{\left(p \beta+q \alpha_{1}\right)^{2} e^{\left(p \beta+q \alpha_{1}\right) T_{2}}}{\left(q \alpha_{1}+p \beta e^{\left(p \beta+q \alpha_{1}\right) T_{2}}\right)^{2}}, & T_{2} \geq 2 \widehat{T},\end{cases}
$$

Then, an immediate launch of product 1 is optimal provided that $\frac{c \gamma}{1-c}<h^{*}$.

The statement of Theorem 3 identifies the sensitivity properties of the optimal launch gap for a given entry time of the competing product. In particular, part (a) of Theorem 3 states that as the expected time of product 2's entry is pushed into the future, it is optimal for brand 1 to respond by increasing the gap $T^{*}$ between launches of competing brands.

While this increase in the launch gap is intuitive, surprisingly, a delay of product 2's launch can drive the optimal launch date for product 1 forward in time. For example, 
consider the case where product 2 is to be launched at time 10 and product 1 at time 2 (the launch gap is 8). Now, product 2 announces a delay in launch, say to time 12 . Depending on the parameters of the problem, it may be optimal for product 1 to respond by moving its launch forward in time (e.g. to launch at time 1), which would lead to a launch gap of 11, or, by moving its launch backward in time (e.g. to launch at time 3), which would lead to a launch gap of 9 . Thus, in both cases, the launch gap is increased, yet the actual launch date for product 1 could go either way.

An illustration of this phenomena is presented in Figure 9: we observe that as the launch time of product 2 increases, the optimal response for product 1 changes discontinuously at $\widehat{T}_{2}=19.5$ from an increasing to a decreasing function. Such discontinuity is a direct consequence of the non-concavity of the product 1 lifecycle sales function $M_{1}(T)$ and occurs when the profit function $P$ has two competing local maxima, so that one of them is preferred for $T_{2}<\widehat{T}_{2}$ and the other one for $T_{2}>\widehat{T}_{2}$. In particular, for $T_{2}<\widehat{T}_{2}$, it is optimal to launch product 1 after product 2, and the optimal launch time for product 1 is an increasing function of $T_{2}$. For $T_{2}>\widehat{T}_{2}$, the optimal market launch response of product 1 changes drastically - it is now optimal to launch product 1 before product 2, and the launch time for product 1 is pushed forward as the launch of a competitor is delayed.

The optimal launch gap is decreasing in the cost parameter $c$. In other words, the better the cost position of product 1 , the more will it benefit from achieving a high level of lifecycle sales (Figure 10a). A decrease in the relative attractiveness of product 2, $\alpha_{2}$, leads to a later launch for product 1 . The intuition behind this result is that the decreased attractiveness of product 2 allows product 1 to spend extra time on cost reduction without incurring significant lifecycle losses (Figure 10b). Figure 10b shows an example of a discontinuous change in $T^{*}$ at $\alpha_{2}^{*}=0.56$. Just like in the example of Figure 7 , such discontinuity reflects an existence of two local maxima in the profit function, with one of them being preferred for $\alpha_{2}<\alpha_{2}^{*}$ and the other for $\alpha_{2}>\alpha_{2}^{*}$. In the example shown in Figure 10b the optimal response for product 1 at $\alpha_{2}=\alpha_{2}^{*}$ exhibits a fundamental change: from launching 6 units of time behind product 2 to launching 10 units ahead. Again, this is a direct result of the non-concavity of lifecycle sales with respect to the launch gap $T$.

Theorem 3 identifies a non-monotone response of the optimal launch gap to changes in the speed of cost reduction $\gamma$. We illustrate this behavior in Figure 11. For small values of $\gamma$, the return to a delay in terms of unit cost reduction is low, thus, the firm will favor a quick launch. In the other extreme, if $\gamma$ is large, i.e. cost reductions are achieved extremely rapidly, not much of a delay is needed. Even a small delay is sufficient to achieve a good cost position. In-between $(\gamma=0.2$ in Figure 11), the delay will be at a maximum, 
represented by a minimum launch gap.

Part b of Theorem 3 states sufficient conditions for the optimality of an immediate launch of product 1 for the most general case of $\alpha_{1}+\alpha_{2} \neq 1, \alpha_{1}+\alpha_{2}<2$ (similar statements can be obtained for the cases of $\alpha_{1}+\alpha_{2}=1$ and $\alpha_{1}+\alpha_{2}=2$ ). Parameter $h^{*}$ describes the effective rate of sales loss for product 1 in a competitive environment in the case when the market introduction of product 1 is delayed. In Theorem 3 , the analysis of the cost-sales trade-off is reduced to a simple comparison between $h^{*}$ and the effective rate of production cost reduction $\frac{c \gamma}{1-c}$. In particular, the statement of the Theorem quantifies the range of values for the rate of process improvement $\gamma$ or potential cost reduction $c$ for which delays in market introduction of a new product are not warranted.

(17) indicates that, as expected, the effective rate of sales loss $h^{*}$ is a decreasing function of the competitor's market entry time $T_{2}$. If the competitor (product 2) has already entered the market or is planning to enter in not-too-distant future (sooner than $2 \widehat{T}$ ), the immediate market entry for product 1 is imperative under innovation-driven category-level demand processes (high values of $p$ and low values of $q$ ). This conclusion is intuitively appealing, since innovation-driven demand processes are 'faster' than imitation-driven ones, characterized by low values of $p$ and high values of $q$ : they start at higher sales rates, reach sales peaks sooner and 'die out' faster. If the competitor's market entry is

expected in a distant future (later than $2 \widehat{T}$ ), the optimality of the immediate launch for the same values of category diffusion parameters is no longer assured: as the expected competitor's entry is shifted into the future, there is an increasing incentive to delay the launch of product 1 and benefit from the production cost savings. The sufficient conditions of Theorem 3 are quite general in that they guarantee the optimality of the immediate launch of product 1 irrespective of the attractiveness level of the competing product 2 .

\section{Competitive Market Entry Decisions}

The analysis of Section 5 focuses on a company's optimal response for a given market entry of a competitor. While such analysis may be valid in the settings when competition has already made its move $\left(T_{2}<0\right)$ or is committed to a certain launch time in the future, an equilibrium analysis of the market entry decisions is generally more desirable. In this Section we consider a setting in which at $t=0$ neither of the competitors have entered the market. Following the notation defined in Section 3, we can express the profit functions of the two competitors as

$$
\Pi_{1}\left(T_{1}, T_{2}\right)=M_{1}\left(T_{2}-T_{1}\right)\left(1-c_{1} e^{-\gamma_{1} T_{1}}\right),
$$




$$
\Pi_{2}\left(T_{1}, T_{2}\right)=\left(m-M_{1}\left(T_{2}-T_{1}\right)\right)\left(1-c_{2} e^{-\gamma_{2} T_{2}}\right)
$$

where we have introduced the cost parameters for the second competitor, $c_{2}$ and $\gamma_{2}$. Our objective is to characterize a Nash equilibrium in market entry times. In equilibrium, each competitor will choose its market entry time as the best possible response to the market entry time of its competitor. Thus, $\left(T_{1}^{*}, T_{2}^{*}\right)$ are an equilibrium if:

$$
\begin{aligned}
& T_{1}^{*}=\arg \max _{T_{1} \geq 0}\left(\Pi_{1}\left(T_{1}, T_{2}^{*}\right)\right), \\
& T_{2}^{*}=\arg \max _{T_{2} \geq 0}\left(\Pi_{2}\left(T_{1}^{*}, T_{2}\right)\right) .
\end{aligned}
$$

The complex structure of the lifecycle sales function $M_{1}(T)$ complicates the equilibrium analysis substantially, both with respect to establishing existence and uniqueness of the solution to (20)-(21). However, a set of general results characterizing $T_{1}^{*}$ and $T_{2}^{*}$ can still be obtained as outlined below.

We first provide a partial characterization of the 'entry' Nash equilibrium in the symmetric case of $\alpha_{1}=\alpha_{2}=\alpha, \beta=0.5, c_{1}=c_{2}=c, \gamma_{1}=\gamma_{2}=\gamma$. For $0.5<\alpha<1$, define

$$
\begin{gathered}
c^{*}=\frac{\frac{p}{2}\left(1+\frac{q}{p}\right)^{2 \alpha-1}}{\gamma+\frac{p}{2}\left(1+\frac{q}{p}\right)^{2 \alpha-1}}, \\
B(c)=1-\frac{q \alpha+\frac{p}{2}}{q \alpha+\frac{p}{2}\left(\frac{c}{c^{*}}\right)^{\frac{q \alpha+\frac{p}{2}}{\gamma}}},
\end{gathered}
$$

and

$$
L\left(c^{*}\right)=\left(c>c^{*} \mid(1-c)\left(1+(B(c))\left(\frac{1+\frac{q}{p}}{1+\frac{q}{p}-\frac{q}{p} B(c)}\right)^{2 \alpha-1}\right)>1-c^{*}\right) .
$$

Then, we can establish the following properties of the entry-times Nash-equilibrium:

\section{Theorem 4a (Symmetric products)}

a) For $c \leq c^{*}$, there exists a pure strategy symmetric equilibrium in which both firms favor an immediate launch, i.e. $T_{1}^{*}=T_{2}^{*}=0$.

b) For $c \in L\left(c^{*}\right)$, there exists no solution to (20) and (21), and thus there exists no pure strategy equilibrium.

The first result of Theorem $4 \mathrm{a}$ implies that the identical products enter the market simultaneously as long as the value of the potential cost gains associated with launch delays is low. It is interesting to note that the maximum value of the cost gains assuring the immediate launch is a 'rapidly' increasing function of $\alpha$. In particular, when the competitive 
diffusion shifts to the category level $(\alpha \rightarrow 0.5), c^{*}$ approaches $\frac{p}{2 \gamma+p}$. On the other hand, when the diffusion 'shifts' to the brand level $(\alpha \rightarrow 1), c^{*}$ approaches, $\frac{q}{2 \gamma+q}-$ a potentially much higher value (assuming that the rate of cost improvements $\gamma$ is not too low).

The second result of Theorem 4a indicates that even for the symmetric case a Nash equilibrium in pure strategies is not guaranteed to exist. We could not provide a concise characterization of the entire region in which $c$ is greater than $c^{*}$. However, we could establish that the pure strategy Nash equilibrium certainly does not exist for some $c$ exceeding $c^{*}$, in particular those in the interval $L\left(c^{*}\right)$.

In the absence of pure strategy equilibria, mixed strategy equilibria that involve randomized strategies could still exist. Although it seems difficult to imagine companies randomizing over such important decisions such as launch times, several points are worth noting. The first relates to the structure of the best response function, which is illustrated by Figure 12. The horizontal axis shows the entry time of the competitor and the vertical axis shows the corresponding best response. Given that the competitor launches at relatively early time $T$, a development team prefers to respond with a launch at $T_{r}>T$. Launching after the competitor allows the team to benefit from lower unit costs while also 'free-riding' on the category diffusion set forth by the competitor.

Interpreting the concept of Nash equilibrium as an iterative application of best response functions, we end up in a scenario, where both players iteratively - at least initially - iteratively delay their launch ${ }^{7}$. However, we observe that for every unit of delay of the competitor's launch, the development team delays its own launch only by about 0.5 time units. This can be seen in Figure 12 by comparing the initial part of the best response function with the identity line. Thus, the process of waiting for the other party to launch does not continue indefinitely: once the hypothetical launch time has been delayed sufficiently into the future, the best response time of the development team 'jumps back', favoring a much earlier launch. In Figure 12, we observe that at $T=16$, the response jumps forward discontinuously. Such jump occurs close to the point where the potential pure strategy equilibrium could be realized, preventing such equilibrium from occurring (the best response functions never cross). In absence of pure strategy equilibria, the continuity of our pay-off function directly implies the existence of a mixed strategy equilibrium (Gliksberg 1952, Dasgupta and Maskin 1986).

Second, as observed by Harsanyi (1973), mixed strategies do not have to be interpreted as randomizations in the sense of tossing coins, but could capture (un-modeled) private

\footnotetext{
${ }^{7}$ Note that - of course - strictly speaking there exists no iterative adjustment in a simultaneous game. The word 'iterative' here is meant to illustrate the nature of the Nash equilibrium concept.
} 
information in the game (see also Vives 1999). The existence of private information is plausible in the case of the launch time decisions, as players are unlikely to be fully informed about the detailed decision situation of each other.

Third, mixed strategy equilibria are rather common in models of economics and marketing. For example, the reader might think of the launch time decision of a firm as a location problem in a differentiated market. The optimal location of a store in a non-uniform market depends upon the location of competing stores, and previous research has pointed to the importance of mixed strategies in such games (Anderson et al. 1992). Similarly, Raju et al. (1990) point to mixed strategies concerning the timing of price promotions.

We now turn to the case of 'asymmetric' products, which is obviously more complicated to analyze. Some general characterization of the 'entry' Nash equilibrium however can still be obtained. Note that if the solution to (20)-(21) exists, then $T_{1}^{*}$ is one of the local maxima of $\Pi_{1}\left(T_{1}, T_{2}^{*}\right)$ and $T_{2}^{*}$ is one of the local maxima of $\Pi_{2}\left(T_{1}^{*}, T_{2}\right)$. Below we denote 'matching' pairs of local maxima of $\Pi_{1}$ and $\Pi_{2}$ as $T_{1}^{l}$ and $T_{2}^{l}$ : $T_{1}^{l}$ is a local maximum of $\Pi_{1}\left(T_{1}, T_{2}^{l}\right)$

and $T_{2}^{l}$ is a local maximum of $\Pi_{1}\left(T_{1}^{l}, T_{2}\right)$. The following result provides a characterization of the properties of local maxima of the competitors' profit functions:

\section{Theorem 4b (non-symmetric products)}

i) Let $\alpha_{1}+\alpha_{2} \neq 1, \alpha_{1}+\alpha_{2}<2$ and define

$$
\begin{aligned}
\theta_{1} & =\frac{p \beta\left(1+\frac{q}{p}\right)^{\alpha_{1}+\alpha_{2}-1}(1-\beta)}{\frac{1-\alpha_{2}}{2-\alpha_{1}-\alpha_{2}}+\frac{p}{q\left(\alpha_{1}+\alpha_{2}-1\right)}\left(\beta-\frac{1-\alpha_{2}}{2-\alpha_{1}-\alpha_{2}}\right)\left(\left(1+\frac{q}{p}\right)^{\alpha_{1}+\alpha_{2}-1}-1\right)}, \\
\theta_{2} & =\frac{p \beta\left(1+\frac{q}{p}\right)^{\alpha_{1}+\alpha_{2}-1}(1-\beta)}{\frac{1-\alpha_{1}}{2-\alpha_{1}-\alpha_{2}}-\frac{p}{q\left(\alpha_{1}+\alpha_{2}-1\right)}\left(\beta-\frac{1-\alpha_{2}}{2-\alpha_{1}-\alpha_{2}}\right)\left(\left(1+\frac{q}{p}\right)^{\alpha_{1}+\alpha_{2}-1}-1\right)} .
\end{aligned}
$$

Then, $T_{1}^{l}=T_{2}^{l}=0 \Leftrightarrow \frac{c_{i} \gamma_{i}}{1-c_{i}} \leq \theta_{i}, i=1,2$.

ii) Let

$$
\begin{aligned}
& \widehat{T}_{1}=\left(-T \mid T \leq 0, \frac{1}{M_{1}} \frac{d M_{1}}{d T}=\frac{c_{1} \gamma_{1} e^{\gamma_{1} T}}{1-c_{1} e^{\gamma_{1} T}}, \frac{d^{2} M_{1}}{d T^{2}} \frac{1-c_{1} e^{\gamma_{1} T}}{1+c_{1} e^{\gamma_{1} T}} \leq \gamma_{1} \frac{d M_{1}}{d T}\right) \\
& \widehat{T}_{2}=\left(T \mid T \geq 0, \frac{1}{m-M_{1}} \frac{d M_{1}}{d T}=\frac{c_{2} \gamma_{2} e^{-\gamma_{2} T}}{1-c_{2} e^{-\gamma_{2} T}},-\frac{d^{2} M_{1}}{d T^{2}} \frac{1-c_{2} e^{-\gamma_{2} T}}{1+c_{2} e^{-\gamma_{2} T}} \leq \gamma_{2} \frac{d M_{1}}{d T}\right) .
\end{aligned}
$$

Then, $T_{1}^{l}=0, T_{2}^{l}=\widehat{T}_{2} \Leftrightarrow \frac{c_{1} \gamma_{1}}{1-c_{1}} \leq \frac{1}{M_{1}\left(\widehat{T}_{2}\right)} \frac{d M_{1}}{d T}\left(\widehat{T}_{2}\right)$. Also, $T_{1}^{l}=\widehat{T}_{1}, T_{2}^{l}=0 \Leftrightarrow \frac{c_{2} \gamma_{2}}{1-c_{2}} \leq$ $\frac{1}{m-M_{1}\left(\widehat{T}_{1}\right)} \frac{d M_{1}}{d T}\left(\widehat{T}_{1}\right)$. 
iii) Let

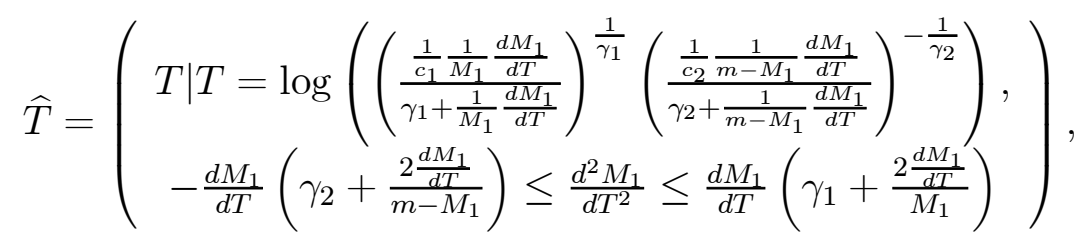

Then,

$$
\begin{aligned}
& T_{1}^{l}=-\frac{1}{\gamma_{1}} \log \left(\frac{\frac{1}{c_{1}} \frac{1}{M_{1}(\widehat{T})} \frac{d M_{1}}{d T}(\widehat{T})}{\gamma_{1}+\frac{1}{M_{1}(\widehat{T})} \frac{d M_{1}}{d T}(\widehat{T})}\right), \\
& T_{2}^{l}=-\frac{1}{\gamma_{2}} \log \left(\frac{\frac{1}{c_{2}} \frac{1}{m-M_{1}(\widehat{T})} \frac{d M_{1}}{d T}(\widehat{T})}{\gamma_{2}+\frac{1}{m-M_{1}(\widehat{T})} \frac{d M_{1}}{d T}(\widehat{T})}\right) .
\end{aligned}
$$

While the statements of part i) of Theorem $4 \mathrm{~b}$ relate to the most general case of $\alpha_{1}+\alpha_{2} \neq 1, \alpha_{1}+\alpha_{2}<2$, similar statements for any other combination of diffusion parameters can be easily obtained.

Several comments are due with respect to this Theorem. First, as indicated by Theorem $4 \mathrm{a}$, the existence of the solutions to $(27),(28)$ or (29) in the case of $\frac{c_{1} \gamma_{1}}{1-c_{1}}>\theta_{1}$ or $\frac{c_{2} \gamma_{2}}{1-c_{2}}>\theta_{2}$ is not guaranteed. Second, the non-concavity of the lifecycle sales $M_{1}(T)$ implies that the uniqueness of the solutions to (27), (28) or (29), if they exist, is also not guaranteed. The complex nature of the interaction between competitors in the diffusion model (4) requires an evaluation of all possibilities outlined in parts i)-iii) of Theorem $4 \mathrm{~b}$ for a given set of problem parameters.

In order to illustrate the various cases of Theorem 4b, we conducted a numerical study for the case of two symmetric products with different cost structures (Table 2) as well as two different products with symmetric cost structures (Table 3).

The results in Table 2 suggest that a pure strategy equilibrium is more likely to exist if the competitive diffusion occurs primarily at the brand level $\left(\alpha_{1}=\alpha_{2}=0.9\right)$ as opposed to the category level. In this case, a completely symmetric set-up will lead to an immediate launch of both competitors. This is consistent with the results of Theorem 4a. Note that the potential cost improvement parameter $c=0.1$ is less than the critical value $c^{*}=\frac{\frac{p}{2}\left(1+\frac{q}{p}\right)^{2 \alpha-1}}{\gamma+\frac{p}{2}\left(1+\frac{q}{p}\right)^{2 \alpha-1}}=0.48$.

For higher values of the innovation parameter, there exists a competitive advantage for one of the competitors. In this case, it becomes beneficial to 'trade' this additional advantage and capture some of the potential cost improvements by delaying launch. We further note that as the diffusion shifts to the category level $\left(\alpha_{1}=\alpha_{2}=0.55\right)$, the pure 
strategy equilibrium becomes impossible to achieve. A similar situation occurs in the case of non-symmetric competing products $\left(\alpha_{1}=0.9, \alpha_{2}=0.55\right)$ unless the products are balanced on the innovation scale. This observation is in agreement with the result of part (i) of Theorem 4b: for the data parameters we consider, setting $\beta$ to 0.5 allows the values of $\theta_{1}$ and $\theta_{2}$ to exceed the respective cost parameter combinations, forcing the competitors to focus exclusively on the market share side of their respective profit functions and, therefore, to launch immediately.

Similar to Table 2, Table 3 illustrates the basic trade-off between potential cost improvements and losses of sales. However, now we focus on the effect of the cost parameters, for a given set of diffusion characteristics, namely the one where consumers purchase at the brand level $\left(\alpha_{1}=\alpha_{2}=0.9\right)$. Pure equilibria exist for a wide range of cost parameters. When the potential cost savings are equally high for both products and can be achieved equally fast $\left(c_{1}=c_{2}=0.5, \gamma_{1}=\gamma_{2}=0.1\right)$, the pure strategy equilibrium will lead to delayed launches for both players. In this case, both products are delayed by the same amount of time $\left(T_{1}^{*}=T_{2}^{*}=0.399\right)$. As cost savings for the second competitor become harder to realize $\left(c_{1}=c_{2}=0.5, \gamma_{1}=0.1, \gamma_{2}=0.001\right)$, the equilibrium shifts to an immediate launch of player 2 . In contrast, player 1 prefers to slightly delay its launch, leading to a better cost position $\left(T_{1}^{*}=0.252, T_{2}^{*}=0\right)$.

\section{$7 \quad$ Practical Implications}

Based on our results analyzing the impact of launch time on a new product's demand dynamics, we can now revisit the question of 'What is the financial impact of a change in launch time on the total profits over the product's lifecycle?'. Recall that such a 'dollarization' of changes in launch time allows a development team to trade-off the four product development performance measures depicted in Figure 1. In this section we identify three ways our results can be used to improve the decision making of a development team.

\section{Shape of profit (launch time) function}

Consider a project team developing a new product, which is planned to launch in August 2003. Holding everything else constant, the team will almost certainly prefer to have the product ready to launch in June 2003. In other words, sales and - holding everything else constant - profits, are increasing with acceleration. While this monotonic effect of acceleration on profits is rather straightforward, the second derivative of this effect is much harder to grasp. Common wisdom, as summarized for example in the graph by Urban and Hauser (see Figure 2) suggests that there exist diminishing returns to acceleration. In 
the above example, this implies that the financial gains of moving the launch time from August 2003 to June 2003 would be larger than the additional gains of moving the launch time from June 2003 to April 2003.

Our results analyzing the impact of launch time on a new product's demand dynamics allow us to analyze the second derivative of this effect explicitly. Consider Figure 13, which plots the impact of acceleration on revenue. Similar to the Urban and Hauser Figure, the horizontal axis shows various levels of speed $\left(40-T_{1}\right)$. A fast launch corresponds to high values on the horizontal axis. The vertical axis plots revenues for product 1 , which we find - not surprisingly - to be increasing with speed. Consider the case where the diffusion occurs primarily at the brand level (high values of $\alpha_{i}$ ). In this case, returns to acceleration are - although steep - diminishing. Note further that this effect is most pronounced at the point of simultaneous market entry, consistent with the statements we reviewed from Wheelwright and Clark above. In contrast, we observe that for products that diffuse primarily at the category level $\left(\alpha_{1}=\alpha_{2}=0.5\right)$, this pattern changes fundamentally. The reason for this is that returns to acceleration are high, if one product is ahead of the other for a sufficient period of time to capture a significant part of the market potential $m$ prior to entry of the follower. If both firms are competing head-to-head, no firm will be able to attract a significant portion of the market prior to the entry of the follower, which leads to a point of no sales increase in the graph in Figure 13.

Second, consider again the situation of a development team working towards a planned launch in August 2003. In January 2003, the development team learns that the other firm pursuing development for a similar product experiences a set-back and will suffer a three-months delay. How should the development team respond? Intuitively, one would say that the competitor's delay eases the time pressure on the team and that some of this 'gain' should be allocated to the other performance measures unit cost, development budget, and design quality. This corresponds to a (slight) delay in launch. However, as we showed in Figure 9, a delay in the competitor's launch time might actually move the optimal launch time forward, even if it comes at the expense of higher unit costs.

\section{Quantitative Results}

In addition to providing qualitative insights, our results can be used for an accurate quantification of the change in profits as well as the change in sales trajectory that come from a change in launch time. The closed form solutions provided by (15) and (16) can easily be imported to a spread-sheet model which enables the development team to evaluate and compare multiple launch time scenarios. This is directly compatible with the four step procedure proposed by Ulrich and Eppinger. An example of this is given in 
Figure 14, which shows - for a given set of demand parameters - three sales trajectories corresponding to three different launch time scenarios. Observe that the Figure is similar to the one proposed by Rosenthal, the horizontal axis captures time while the vertical axis indicates per period revenues.

Now that we have a coherent model of demand dynamics, we can also analyze other scenarios not considered by Rosenthal, including the case when the competitor's product has already been launched and the case where products differ in terms of their desirability. For example, Figure 14a shows that product 1 can continue its increase in sales beyond the entry of product 2 , as product 1 benefits from its strong word-of-mouth at the brand level $\left(\alpha_{1}=0.9\right)$.

We also observe that as long as the word-of-mouth happens primarily at the brand level, the market leader is only moderately impacted by the beginning of the competitive period. Figure 14b shows an example in which the pioneering product is of lower quality. The moment the second product enters, its sales start to drop, which is consistent with Rosenthal's prediction.

\section{Structural Result: Applicability of trade-off rules}

While the previous two improvements enable development teams to more accurately understand the financial consequences of a changed launch time, leading to refined tradeoff rules, the third improvement questions the usage of trade-off rules per-se. Again, consider a development team working towards a planned launch in August 2003. The team debates if the launch should be delayed to October 2003. The extra two months would reduce product unit costs, which - over the product lifecycle - accumulates to a saving of $\$ 500 \mathrm{k}$. At the same time, the delay would reduce lifecycle sales by $\$ 1$ million. Note that these numbers already assume that the development correctly calculates the impact an October 2003 launch would have on the sales trajectory. Should the launch be delayed?

Under the assumption of diminishing returns to acceleration (e.g. Urban and Hauser), the answer is a profound 'No'. As long as the second derivatives as depicted in Figure 13 are 'well-behaved', a marginal analysis of profits leads the development team to the profit optimal launch time. However, given the more complicated curvature established in this paper, a development team might end-up in a local profit maximum, not realizing that higher profits are obtainable if the launch is altered more substantially. An example of this is provided by Figure 15, which shows a locally optimal solution at $T_{1}=12$ and a second point with zero derivative at $T_{1}=24$.

The first local optimum captures the point in which product 1 enters the market first. 
Delaying market entry further shortens the period product 1 is alone on the market, accelerating (launching before 12) would increase unit costs even further. The second local optimum corresponds to the case where product 1 is a low-cost follower. The local optimality of this point reflects that a further delay of entry would leave product 1 with a sufficiently long monopoly period to capture most of the market. On the other hand, launching earlier would increase unit costs. We observe based on Figure 15 that the profitability of the second optimum decreases with the attractiveness of the competing product.

The existence of several competing local optima is especially important given the cross-

functional nature of product development. Product development decisions typically need to balance the views of various organizational groups involved in the project. In our example above, the marketing representatives on the development team might advocate an accelerated launch of April 2003. In contrast, the engineering representative might request a delay to October 2003. A project manager attempting to find a reasonable compromise between these different proposals is likely to choose some launch time in between, which - in light of Figure 15 (the one with the two profit maxima) - might not be a good choice.

\section{Discussion and Conclusion}

We have started this manuscript with the question 'what is the cost of a one day launch delay?' The literature contains a rather diverse set of qualitative approaches to this problem. While all approaches seem 'intuitively correct', a scientific debate on which one of them is appropriate for a given managerial situation is impossible without an explicit list of underlying assumptions. Especially, the question of how a delayed launch affects the demand dynamics of the new product is hard to answer without specifying a formal model of market demand.

We have presented a detailed demand model of brand-level competition within a Basstype category diffusion. Our model includes a market saturation effect and word-of-mouth effects at both the category and the brand level. We also explicitly capture the "cross wordof-mouth effects' between different brands, which allows us model differences in product attractiveness. This is the most general demand model that has been used for a normative analysis of entry timing in competitive new product diffusion and it matches the current state-of-the-art of empirical research (see Table 1).

In this general setting, we provide analytical results for both products's sales trajectories 
as well as their lifecycle sales (Theorem 1). We use these results to study the impact of launch time delay (acceleration) on overall lifecycle sales (Theorem 2). While at the aggregate level, these results show a remarkable similarity to the qualitative arguments of the existing literature (Figure 2), we show that our analytical findings have direct practical implications for product development decision making, especially with respect to the coordination between marketing and operations (Theorem 3). We also use our results to study the resulting game between two brands choosing their launch times strategically (Theorem 4).

Our analysis related to the cost of delayed product launch opens up several opportunities for future research. First, our model of competition in launch times could be extended to include price competition between the two brands. This would combine our work with earlier research by Eliashberg and Jeuland (1986) as well as Krishnan et al. (1999). Second, the focus of our analysis was on the trade-off between unit production costs and sales revenues. As discussed above, a similar trade-off exists between product performance, and thus ultimately product attractiveness, and sales revenues. For example Kouvelis and Mukhopadhyay (1999) include a control variable about the design quality of the product. Third, our model opens the door for empirical research, which could estimate our measures of product attractiveness. In current models, this effect is frequently compounded with the timing of market introduction, which makes it hard to determine if a newly introduced brand failed because the incumbent brand had already gained sufficient market share or because of superior design quality.

In summary, we believe that more research is needed to quantify trade-offs between product development performance metrics, moving from 'intuitive' arguments and ad-hoc decision rules to more formal and transparent scientific reasoning ${ }^{8}$.

\section{References}

Anderson, S., de Palma, A., and Thisse, J.F., Discrete Choice Theory of Product Differentiation, The MIT Press, Cambridge, MA, 1992.

Bass, F. M. 'A new product growth model for consumer durables', Management Science, Vol. 15, No. 5, 215-227, 1969

Bass, F. M., T. V. Krishnan, D. C. Jain,'Why the Bass Model Fits without Decision Variables', Marketing Science. Vol. 13, No. 3, 203-223, 1994.

Bass, F. M., T. V. Krishnan, D. C. Jain,'Modeling the Marketing-Mix Influence in New-

\footnotetext{
${ }^{8}$ The authors are grateful for comments and suggestions by Christophe Van den Bulte and Jehushua Eliashberg with respect to product diffusion models. We also thank Serguei Netessine and Gerard Cachon for sharing their knowledge on equilibrium analysis.
} 
Product Diffusion', in V. Mahajan, E. Muller, and Y. Wind (eds), New Product Diffusion Models, Kluwer 2000.

Bayus, B. L., S. Jain, and A. G. Rao, 'Too little, too early: Introduction timing and new product performance in the personal digital assistant industry', Journal of Marketing Research, Vol. 34, No. 1,50-63, 1997

Bayus, B. L., N. Kim, A. D. Shocker,'Growth Models for Multiproduct Interactions: Current Status and New Directions', in V. Mahajan, E. Muller, and Y. Wind (eds), New Product Diffusion Models, Kluwer 2000.

Bhattacharya, S., V. Krishnan, and V. Mahajan, 'Managing new product definition in highly dynamic environments', Management Science, Vol. 44, No. 11, S50-S64, 1998

Chatterjee, R., J. Eliashberg, and V. R. Rao, 'Competitive Diffusion Models', presented at the New Product Diffusion Conference, Philadelhia 1998.

Clark, K. 'Project Scope and Project Performance: The Effect of Parts Strategy and Supplier Involvement on Product Development', Management Science, Vol. 35, No. 10, 1247-1263, 1989

Cohen, M. A., J. Eliashberg, T. H. Ho,'An Analysis of Several New Product Performance Metrics', Manufacturing \& Service Operations Management, Vol. 2, No. 4, 337-349, 2000

Dasgupta, P., E. Maskin, 'The Existence of Equilibrium Points in Discontinuous Economic Games, I and II', Review of Economic Studies, Vol. 53, No. 2, 1-41, 1986

De Groote, X., 'Flexibility and marketing/manufacturing coordination', International Journal of Production Economics, Vol. 36, No. 2, 153-167, 1994

Eliashberg, J., and A. P. Jeuland, 'The Impact of Competitive Entry in a Developing Market upon Dynamic Pricing Strategies', Marketing Science. Vol. 5, No. 1, 20-36. 1986

Eliashberg, J., D. Manceau, V. Rao, 'A New Diffusion Model for Pre-announced Products', Working Paper, The Wharton School, 2002.

Fershtman, C., V. Mahajan, and E. Muller, 'Advertising, Pricing and Stability in Oligopolistic Markets', Working Paper, Northwestern University, 1983

Gliksberg, I. L.,'A further Generalization of the Kakutani Fixed Point Theorem with Application to Nash Equilibrium Points', Proceedings of the American Mathematical Society, Vol. 38, 170-174, 1952.

Harsanyi, J. C., 'Games with Randomly Distributed Pay-offs: A New Rationale for Mixed-Strategy Equilibrium Points', International Journal of Game Theory, Vol. 1, 1-25, 1973 
Kalish, S. and G. L. Lilien, 'A Market Entry Timing Model for New Technologies', Management Science, Vol. 32, No. 2, 194-205, 1986

Kalyanaram, G. and V. Krishnan, 'Deliberate product definition: Customizing the product definition process', Journal of Marketing Research, Vol. 34, No. 2, 276-285, 1997.

Krishnan, T. V., F. M. Bass, D. C. Jain, 'Optimal Pricing Strategy for New Products', Management Science, Vol. 45, No. 12, 1650-1663, 1999

Kouvelis, P. and S. Mukhopadhyay, 'Modeling the design quality competition for durable products', IIE Transactions, Vol. 31, No. 9, 865-880, 1999

Krishnan, T. V., F. M. Bass, and V. Kumar, 'Impact of a late entrant on the diffusion of a new product/service'. Journal of Marketing Research. Vol. 37, No. 2, 269-278, 2000.

Mahajan, V., S. Sharma, R. D. Buzzell, 'Assessing the impact of competitive entry on market expansion and incumbent sales', Journal of Marketing, Vol. 57, No. 3, 39-52, 1993.

Mansfield, E. , 'The importance of price elasticity of demand', Managerial Economics of Operations Research, 3d edition, W.W. Norton and Co., 1975.

Peterson, R. and V. Mahajan,'Multi Product Growth Models', in J. Sheth (ed), Research in Marketing, JAI Press, 1978.

Porter, M., Competitive Advantage: Creating and Sustaining Superior Performance, Free Press 1985.

Raju, J. S., V. Srinivasan, R. Lal,'Brand Loyalty and Competitive Price Promotion Strategy', Management Science, Vol. 36, No. 3, 276-304, 1990

Rosenthal, S. R., Effective Product Design and Development, Irwin, 1992.

Schmidt, G. M., E. L. Porteus,'The Impact of an Integrated Marketing and Manufacturing Innovation', Manufacturing 85 Service Operations Management, Vol. 2, No. 4, $317-336,2000$

Smith, P. G and D. G. Reinertsen, Developing Products in Half the Time, John Wiley \& Sons 1991.

Ulrich, K. and S. Eppinger, Product Design and Development, McGraw Hill, 1999.

Urban, G. L. and J. R. Hauser, Design and Marketing of New Products, Prentice Hall, 1993.

Van den Bulte, C. 'New Product Acceleration: Measurement and Analysis,' Marketing Science, 19, 4, 366-380, 2000

Vives, X., Oligopoly Pricing: Old Ideas and New Tools, MIT Press, 1999.

Wheelwright, S. and K. Clark, Revolutionizing Product Development, Free Press, 1992. 
Xie, J., X. M. Song, M. Sirbu, Q. Wang,'Kalman Filter Estimation of New Product Diffusion Models', Journal of Marketing Research, Vol. 34, No. 3, 378-393, 1997.

\section{Appendix}

\section{Proof of Lemma 1}

In our proof we only consider the case of $T<0$, since the proof for $T>0$ follows similar steps. For $T<0$ product 2 follows Bass-like diffusion for a period of time equal to $T$ before the first sales of product 1 occur at $t=t_{\mathrm{c}}$. By that time, the cumulative sales

of product 2 reach the level of $\bar{D}_{2}(T)$. Thus, for $t>t_{\mathrm{c}}$ the competitive diffusion process proceeds according to (1) subject to initial conditions $D_{1}\left(t=t_{\mathrm{c}}\right)=0, D_{2}\left(t=t_{\mathrm{c}}\right)=\bar{D}_{2}(T)$. Consider an 'adjusted' sales function $\bar{D}_{2}(\bar{t})=D_{2}(\bar{t})-\bar{D}_{2}(T)$, where $\bar{t}=t-t_{\mathrm{c}}$ : for $\bar{t} \geq 0$ (1) becomes

$$
\begin{aligned}
& \frac{d D_{1}}{d \bar{t}}=\left(\bar{p}_{1}(T)+\frac{1}{\bar{m}(T)}\left(\bar{q}_{11}(T) D_{1}(\bar{t})+\bar{q}_{12}(T) \bar{D}_{2}(\bar{t})\right)\right)\left(\bar{m}(T)-D_{1}(\bar{t})-\bar{D}_{2}(\bar{t})\right), \\
& \frac{d \bar{D}_{2}}{d \bar{t}}=\left(\bar{p}_{2}(T)+\frac{1}{\bar{m}(T)}\left(\bar{q}_{21}(T) D_{1}(\bar{t})+\bar{q}_{22}(T) \bar{D}_{2}(\bar{t})\right)\right)\left(\bar{m}(T)-D_{1}(\bar{t})-\bar{D}_{2}(\bar{t})\right)
\end{aligned}
$$

with initial conditions $D_{1}(\bar{t}=0)=\bar{D}_{2}(\bar{t}=0)=0$, where $\bar{m}(T), \bar{p}_{i}(T)$, and $\bar{q}_{i j}(T)$ are given by (11), (12), and (13), respectively.

\section{Proof of Theorem 1}

In the case when competing products enter the market simultaneously $(T=0)$, we use the system of diffusion equations (4) for $t \geq t_{\mathrm{c}}$ to get

$$
\frac{d D_{1}}{d t}=\left(p \beta+\frac{q}{m}\left(\left(\alpha_{1}+\alpha_{2}-1\right) D_{1}(t)+\left(1-\alpha_{2}\right) D(t)\right)\right)(m-D) .
$$

Then, using Bass equation for the category sales $D(t)$, we obtain

$$
\frac{d D_{1}}{d D}=\frac{p \beta+\frac{q}{m}\left(\left(\alpha_{1}+\alpha_{2}-1\right) D_{1}+\left(1-\alpha_{2}\right) D\right)}{p+\frac{q}{m} D} .
$$

Below we focus on the only non-trivial case $0<\alpha_{1}+\alpha_{2}<2, \alpha_{1}+\alpha_{2} \neq 1$. We define $\bar{D}_{1}=D_{1}+\frac{p m\left(\beta+\alpha_{2}-1\right)}{q\left(\alpha_{1}+\alpha_{2}-1\right)}, \bar{D}=D+\frac{p m}{q}$, to obtain

$$
\frac{d \bar{D}_{1}}{d \bar{D}}=\left(\alpha_{1}+\alpha_{2}-1\right) \frac{\bar{D}_{1}}{\bar{D}}+1-\alpha_{2}
$$

Introducing $u(\bar{D})=\frac{\bar{D}_{1}}{\bar{D}}$, we can re-express $(35)$ as

$$
u^{\prime} \bar{D}=\left(\alpha_{1}+\alpha_{2}-2\right) u+1-\alpha_{2}
$$


which can be integrated with the initial conditions $D_{1}\left(t_{\mathrm{c}}\right)=D_{2}\left(t_{\mathrm{c}}\right)=0$ to yield

$$
D_{1}=\frac{p m}{q\left(\alpha_{1}+\alpha_{2}-1\right)}\left(\beta-\frac{\left(1-\alpha_{2}\right)}{\left(2-\alpha_{1}-\alpha_{2}\right)}\right)\left(\left(1+\frac{q}{p m} D\right)^{\alpha_{1}+\alpha_{2}-1}-1\right)+\frac{\left(1-\alpha_{2}\right)}{\left(2-\alpha_{1}-\alpha_{2}\right)} D
$$

In the case of the delay in the market entry between the two products, we apply the transformation of Lemma 1 to (37) to obtain (15). The expressions for the lifecycle sales for both products (16) are immediately obtained by substituting $m$ for $D(\bar{t})$ in $(15)$.

\section{Proof of Theorem 2}

In our analysis of (16) we focus on the case of $T>0, \alpha_{1}+\alpha_{2}<2, \alpha_{1}+\alpha_{2} \neq 1$, since the proofs for the remaining cases are easily obtained by following similar steps.

For $T>0,(16)$ becomes (here we omit the implied dependence on $T$ )

$$
\begin{aligned}
M_{1}= & \left(\frac{\left(1-\alpha_{1}\right) \bar{D}}{2-\alpha_{1}-\alpha_{2}}+\frac{p m}{q\left(\alpha_{1}+\alpha_{2}-1\right)}\left(\beta-\frac{\left(1-\alpha_{2}\right)}{\left(2-\alpha_{1}-\alpha_{2}\right)}\right)\right)\left(\frac{1+\frac{q}{p}}{1+\frac{q \bar{D}}{p m}}\right)^{\alpha_{1}+\alpha_{2}-1} \\
& +\frac{\left(1-\alpha_{2}\right)}{\left(2-\alpha_{1}-\alpha_{2}\right)} m-\frac{p m}{q\left(\alpha_{1}+\alpha_{2}-1\right)}\left(\beta-\frac{\left(1-\alpha_{2}\right)}{\left(2-\alpha_{1}-\alpha_{2}\right)}\right)
\end{aligned}
$$

Differentiating (38) with respect to $\bar{D}$ yields

$$
\frac{\partial M_{1}}{\partial \bar{D}}=\frac{\left(1+\frac{q}{p}\right)^{\alpha_{1}+\alpha_{2}-1}}{\left(1+\frac{q \bar{D}}{p m}\right)^{\alpha_{1}+\alpha_{2}}}\left(1-\beta+\frac{q\left(1-\alpha_{1}\right) \bar{D}}{p m}\right)>0
$$

As a result, $\frac{d M_{1}}{d T}=\frac{\partial M_{1}}{\partial \bar{D}} \frac{\partial \bar{D}}{\partial T}>0$. Further, differentiating (38) with respect to $\beta$, we get

$$
\frac{\partial M_{1}}{\partial \beta}=\frac{p m}{q\left(\alpha_{1}+\alpha_{2}-1\right)}\left(\left(\frac{1+\frac{q}{p}}{1+\frac{q \bar{D}}{p m}}\right)^{\alpha_{1}+\alpha_{2}-1}-1\right)+\frac{\partial M_{1}}{\partial \bar{D}} \frac{\partial \bar{D}}{\partial \beta}>0
$$

since both addends on the right-hand side of (40) are positive.

The easiest way to show the monotonicity of $M_{1}$ with respect to $\alpha_{1}$ is by using the original diffusion equations. Consider changing $\alpha_{1}$ to $\alpha_{1}+\delta \alpha$ and define

$$
f_{1}(t)=\lim _{\delta \alpha \rightarrow 0}\left(\frac{D_{1}(t, \alpha+\delta \alpha)-D_{1}(t, \alpha)}{\delta \alpha}\right) .
$$

Subtracting product 1 diffusion equation for $\alpha_{1}+\delta \alpha$ from that one for $\alpha_{1}$, dividing the difference by $\delta \alpha$, and taking the limit of $\delta \alpha \rightarrow 0$, we get a differential equation for $f_{1}(t)$ :

$$
\frac{d f_{1}(t)}{d t}=\frac{q}{m}(m-D)\left(\alpha_{1}+\alpha_{2}-1\right) f_{1}(t)+\frac{q}{m}(m-D) D_{1},
$$


with the initial condition $f_{1}(t=0)=0$. Let us define $t_{0}=\min (t>0, f(t)>0)$. From the initial condition on $f_{1}(t)$ and the non-negativity of $\frac{q}{m}(m-D) D_{1}$ it follows that $f_{1}(t)=0$ for all $t \in\left[0, t_{0}\right)$. Then, $\frac{d f_{1}\left(t=t_{0}-0\right)}{d t}>0$, and $f_{1}(t)$ remains positive for some finite interval after $t_{0}$. After that, $f_{1}(t)$ cannot return to 0 due to the presence of the term $\frac{q}{m}(m-D) D_{1}$ in (42). Thus, $f_{1}(t) \geq 0$ for all $t \geq t_{0}$, and, consequently, $\frac{D_{1}(t, \alpha+\delta \alpha)-D_{1}(t, \alpha)}{\delta \alpha}>0$ for small enough $\delta \alpha$. As a result, $\frac{\partial M_{1}}{\partial \alpha_{1}}>0$. Similarly, $\frac{\partial M_{2}}{\partial \alpha_{2}}>0$, so that $\frac{\partial M_{1}}{\partial \alpha_{2}}=\frac{\partial\left(m-M_{2}\right)}{\partial \alpha_{1}}<0$.

For the second-order properties of $M_{1}$ we get

$$
\frac{d^{2} M_{1}}{d T^{2}}=\left(\frac{d \bar{D}}{d T}\right)^{2} \frac{d M_{1}}{d \bar{D}}\left(\frac{d}{d \bar{D}}\left(\log \left(\frac{d M_{1}}{d \bar{D}}\right)\right)+\frac{1}{\frac{d \bar{D}}{d T}} \frac{d}{d T}\left(\log \left(\frac{d \bar{D}}{d T}\right)\right)\right)
$$

so that the sign of $\frac{d^{2} M_{1}}{d T^{2}}$ is the same as the sign of

$$
\begin{aligned}
f(\bar{D}) & =m\left(\frac{d}{d \bar{D}}\left(\log \left(\frac{d M_{1}}{d \bar{D}}\right)\right)+\frac{1}{\frac{d \bar{D}}{d T}} \frac{d}{d T}\left(\log \left(\frac{d \bar{D}}{d T}\right)\right)\right) \\
& =\frac{1}{\frac{p(1-\beta)}{q\left(1-\alpha_{1}\right)}+\bar{Y}}-\frac{\alpha_{1}+\alpha_{2}}{\frac{p}{q}+\bar{Y}}+\frac{1}{\frac{p \beta}{q \alpha_{1}}+\bar{Y}}-\frac{1}{1-\bar{Y}},
\end{aligned}
$$

where $\bar{Y}=\frac{\bar{D}}{m}$. In (44) we have used (39) and the fact that $\frac{d \bar{D}}{d T}=\left(p \beta+\frac{q \alpha_{1}}{m} \bar{D}\right)(m-\bar{D})$ for $T>0$. Further, define

$$
\begin{aligned}
A_{0}= & \left(\frac{p}{q}\right)^{2}\left(\frac{1-\beta}{1-\alpha_{1}}+\frac{\beta}{\alpha_{1}}-\frac{\left(\alpha_{1}+\alpha_{2}+\frac{p}{q}\right) \beta(1-\beta)}{\alpha_{1}\left(1-\alpha_{1}\right)}\right) \\
A_{1}= & \frac{p}{q}\left(\left(1-\alpha_{1}-\alpha_{2}\right)\left(\frac{1-\beta}{1-\alpha_{1}}+\frac{\beta}{\alpha_{1}}\right)+2\right) \\
& +\left(\frac{p}{q}\right)^{2}\left(\frac{2\left(\alpha_{1}+\alpha_{2}\right) \beta(1-\beta)}{\alpha_{1}\left(1-\alpha_{1}\right)}+\left(\alpha_{1}+\alpha_{2}-1\right)\left(\frac{1-\beta}{1-\alpha_{1}}+\frac{\beta}{\alpha_{1}}\right)\right) \\
A_{2}= & 2-\alpha_{1}-\alpha_{2}+\frac{p}{q}\left(\left(\alpha_{1}+\alpha_{2}-1\right)\left(\frac{1-\beta}{1-\alpha_{1}}+\frac{\beta}{\alpha_{1}}\right)-2\right), A_{3}=\alpha_{1}+\alpha_{2}-3,
\end{aligned}
$$

and let

$$
\begin{aligned}
& \bar{Y}_{\min }=\min \left(Y \mid A_{3} Y^{3}+A_{2} Y^{2}+A_{1} Y+A_{0}=0,0 \leq Y \leq 1\right) \\
& \bar{Y}_{\max }=\max \left(Y \mid A_{3} Y^{3}+A_{2} Y^{2}+A_{1} Y+A_{0}=0,0 \leq Y \leq 1\right)
\end{aligned}
$$

Re-arranging terms in (44), we obtain

$$
f(\bar{D})=\frac{A_{3}(\bar{Y})^{3}+A_{2}(\bar{Y})^{2}+A_{1} \bar{Y}+A_{0}}{\left(\frac{p(1-\beta)}{q\left(1-\alpha_{1}\right)}+\bar{Y}\right)\left(\frac{p}{q}+\bar{Y}\right)\left(\frac{p \beta}{q \alpha_{1}}+\bar{Y}\right)(1-\bar{Y})}
$$


with $A_{i}, i=0, . ., 3$, defined in (45). We note that $\sum_{i=0}^{3} A_{i}<0$, and, thus, $f(\bar{D})<0$ for $\bar{Y}>\bar{Y}_{\max }$. Finally, the sign of $f$ for $\bar{Y}<\bar{Y}_{\min }$ is determined by the sign of $A_{0}$. The connection between $\bar{T}$ and $\bar{Y}$ is established from (6), so that

$$
\bar{T}_{\min }=\frac{1}{p \beta+q \alpha_{1}} \log \left(\frac{1-\frac{q \alpha_{1} \bar{Y}_{\min }}{p \beta}}{1-\bar{Y}_{\min }}\right), \bar{T}_{\max }=\frac{1}{p \beta+q \alpha_{1}} \log \left(\frac{1-\frac{q \alpha_{1} \bar{Y}_{\max }}{p \beta}}{1-\bar{Y}_{\max }}\right) .
$$

\section{Proof of Theorem 3}

The the optimal solution to (10) is given by of the critical points of the profit function $M_{1}(T)\left(1-c e^{-\gamma\left(T_{2}-T\right)}\right)$ or by $T_{2}$. Differentiating the profit function with respect to $T$, we obtain an equation for the set $S$ of profit's critical points

$$
\gamma\left(1-\frac{1}{1-c e^{\gamma\left(T-T_{2}\right)}}\right)+\frac{d \log \left(M_{1}(T)\right)}{d T}=0
$$

where $M_{1}$ is expressed by (16). Let $S_{\mathrm{c}}$ be a subset of $S$ such that for every $T_{\mathrm{c}} \in S_{\mathrm{c}}$

$$
\frac{-c \gamma^{2} e^{\gamma\left(T_{\mathrm{c}}-T_{2}\right)}}{\left(1-c e^{\gamma\left(T_{\mathrm{c}}-T_{2}\right)}\right)^{2}}+\frac{d^{2} \log \left(M_{1}\left(T_{\mathrm{c}}\right)\right)}{d\left(T_{\mathrm{c}}\right)^{2}}<0 .
$$

$S_{\text {c }}$ represents a set of local maxima of the profit function. Then, the optimal launch gap for product $1, T^{*}$ is

$$
T^{*}=\arg \max _{T \in\left\{S_{\mathrm{c}} \cup T_{2}\right\}}\left(\left(1-c e^{\gamma\left(T-T_{2}\right)}\right) M_{1}(T)\right) .
$$

Clearly, in order to proof the statement in a), it is sufficient to consider the sensitivity properties of the critical points $T_{\mathrm{c}}$. Differentiating (49) with respect to $c$, we get

$$
\frac{\partial T_{\mathrm{c}}}{\partial c}=\frac{\frac{\gamma e^{\gamma\left(T_{\mathrm{c}}-T_{2}\right)}}{\left(1-c e^{\left.\gamma\left(T_{\mathrm{c}}-T_{2}\right)\right)^{2}}\right.}}{\left(\frac{-c \gamma^{2} e^{\gamma\left(T_{\mathrm{c}}-T_{2}\right)}}{\left(1-c e^{\gamma\left(T_{\mathrm{c}}-T_{2}\right)}\right)^{2}}+\frac{d^{2} \log \left(M_{1}\left(T_{\mathrm{c}}\right)\right)}{d\left(T_{\mathrm{c}}\right)^{2}}\right)}<0,
$$

as the numerator in (49) is positive and the denominator is negative, according to (50). Similarly,

$$
\frac{\partial T_{\mathrm{c}}}{\partial T_{2}}=-\frac{\frac{c \gamma^{2} e^{\gamma\left(T_{\mathrm{c}}-T_{2}\right)}}{\left(1-c e^{\left.\gamma\left(T_{\mathrm{c}}-T_{2}\right)\right)^{2}}\right.}}{\left(\frac{-c \gamma^{2} e^{\gamma\left(T_{\mathrm{c}}-T_{2}\right)}}{\left(1-c e^{\gamma\left(T_{\mathrm{c}}-T_{2}\right)}\right)^{2}}+\frac{d^{2} \log \left(M_{1}\left(T_{\mathrm{c}}\right)\right)}{d\left(T_{\mathrm{c}}\right)^{2}}\right)}>0,
$$

For the derivative with respect to $\alpha_{2}$ we obtain

$$
\frac{\partial T_{\mathrm{c}}}{\partial \alpha_{2}}=-\frac{\frac{\partial}{\partial \alpha_{2}}\left(\frac{d \log \left(M_{1}\left(T_{\mathrm{c}}\right)\right)}{d T_{\mathrm{c}}}\right)}{\left(\frac{-c \gamma^{2} e^{\gamma\left(T_{\mathrm{c}}-T_{2}\right)}}{\left(1-c e^{\gamma\left(T_{\mathrm{c}}-T_{2}\right)}\right)^{2}}+\frac{d^{2} \log \left(M_{1}\left(T_{\mathrm{c}}\right)\right)}{d\left(T_{\mathrm{c}}\right)^{2}}\right)} .
$$


so that the sign of $\frac{\partial T_{\mathrm{c}}}{\partial \alpha_{2}}$ coincides with the sign of $\frac{\partial}{\partial \alpha_{2}}\left(\frac{d \log \left(M_{1}\left(T_{\mathrm{c}}\right)\right)}{d T_{\mathrm{c}}}\right)$. Below we analyze the case $T>0$ (the proof for the case $T<0$ follows similar steps). We note that

$$
\frac{d M_{1}\left(T_{\mathrm{c}}\right)}{d T_{\mathrm{c}}}=\frac{\left(1+\frac{q}{p}\right)^{\alpha_{1}+\alpha_{2}-1}}{\left(1+\frac{q \bar{D}}{p m}\right)^{\alpha_{1}+\alpha_{2}}}\left(1-\beta+\frac{q\left(1-\alpha_{1}\right) \bar{D}}{p m}\right) \frac{d \bar{D}\left(T_{c}\right)}{d T_{\mathrm{c}}}
$$

and, that for $T>0, \frac{\partial \bar{D}\left(T_{c}\right)}{\partial \alpha_{2}}=0$. The sign of $\frac{\partial}{\partial \alpha_{2}}\left(\frac{d M_{1}\left(T_{\mathrm{c}}\right)}{d T_{\mathrm{c}}}\right)$ coincides with the sign of $\frac{\partial}{\partial \alpha_{2}}\left(\log \left(\frac{d M_{1}\left(T_{\mathrm{c}}\right)}{d T_{\mathrm{c}}}\right)\right)$, and

$$
\frac{\partial}{\partial \alpha_{2}}\left(\log \left(\frac{d M_{1}\left(T_{\mathrm{c}}\right)}{d T_{\mathrm{c}}}\right)\right)=\log \left(\frac{1+\frac{q}{p}}{1+\frac{q \bar{D}}{p m}}\right)>0
$$

Theorem 2 states that $\frac{\partial M_{1}}{\partial \alpha_{2}}<0$, so that

$$
\frac{\partial}{\partial \alpha_{2}}\left(\frac{d \log \left(M_{1}\left(T_{\mathrm{c}}\right)\right)}{d T_{\mathrm{c}}}\right)=-\frac{1}{\left(M_{1}\left(T_{\mathrm{c}}\right)\right)^{2}} \frac{\partial M_{1}}{\partial \alpha_{2}} \frac{d M_{1}\left(T_{\mathrm{c}}\right)}{d T_{\mathrm{c}}}+\frac{1}{M_{1}\left(T_{\mathrm{c}}\right)} \frac{\partial}{\partial \alpha_{2}}\left(\frac{d M_{1}\left(T_{\mathrm{c}}\right)}{d T_{\mathrm{c}}}\right)>0
$$

and $\frac{\partial T_{\mathrm{c}}}{\partial \alpha_{2}}>0$. Further,

$$
\frac{\partial T_{\mathrm{c}}}{\partial \gamma}=-\frac{\left(\frac{c e^{\gamma\left(T_{\mathrm{c}}-T_{2}\right)}}{1-c e^{\gamma\left(T_{\mathrm{c}}-T_{2}\right)}}\right)\left(-1+\frac{\gamma\left(T_{2}-T_{\mathrm{c}}\right)}{1-c e^{-\gamma\left(T_{2}-T_{\mathrm{c}}\right)}}\right)}{\left(\frac{-c \gamma e^{\gamma\left(T_{\mathrm{c}}-T_{2}\right)}}{\left(1-c e^{\gamma\left(T_{\mathrm{c}}-T_{2}\right)}\right)^{2}}+\frac{d^{2} \log \left(M_{1}\left(T_{\mathrm{c}}\right)\right)}{d\left(T_{\mathrm{c}}\right)^{2}}\right)}
$$

so that $\frac{\partial T_{\mathrm{c}}}{\partial \gamma}>0$ when $\frac{\gamma\left(T_{2}-T_{\mathrm{c}}\right)}{1-c e^{-\gamma\left(T_{2}-T_{\mathrm{c}}\right)}}>1$ and $\frac{\partial T_{\mathrm{c}}}{\partial \gamma}<0$ when $\frac{\gamma\left(T_{2}-T_{\mathrm{c}}\right)}{1-c e^{-\gamma\left(T_{2}-T_{\mathrm{c}}\right)}}<1$. Letting $x=$ $\gamma\left(T_{2}-T_{\mathrm{c}}\right)$ and defining $x(c)$ as the solution to $(1-x) e^{x}=c$, we observe that $\frac{\partial T_{\mathrm{c}}}{\partial \gamma}<0$ is equivalent to $x<x(c)$. Further, for $\gamma=0, T_{\mathrm{c}}$ equals to $T_{2}$, since in that case (10) reduces to maximization of $M_{1}(T)$. Then, for small $\gamma, \frac{\partial T_{\mathrm{c}}}{\partial \gamma}<0$ and $\frac{\partial\left(\gamma\left(T_{2}-T_{\mathrm{c}}\right)\right)}{\partial \gamma}>0$. As $\gamma$ grows, the inequality $\frac{\partial T_{\mathrm{c}}}{\partial \gamma}<0$ remains valid for $\gamma \leq \gamma^{*}=\min \left(\gamma \mid \gamma\left(T_{2}-T^{*}\right)=x(c)\right)$. At $\gamma^{*}$, $\frac{\partial T_{\mathrm{c}}}{\partial \gamma}=0$, and $\frac{\partial\left(\gamma\left(T_{2}-T_{\mathrm{c}}\right)\right)}{\partial \gamma}=\left(T_{2}-T_{\mathrm{c}}\right)>0$, so that $\frac{\partial T_{\mathrm{c}}}{\partial \gamma} \geq 0$ for $\gamma$ slightly above $\gamma^{*}$. It is clear that if, at any greater value of $\gamma, \gamma\left(T_{2}-T_{\mathrm{c}}\right)$ again becomes equal to $x(c)$, then, for that value of $\gamma, \frac{\partial T_{\mathrm{c}}}{\partial \gamma}=0$, and $\frac{\partial\left(\gamma\left(T_{2}-T_{\mathrm{c}}\right)\right)}{\partial \gamma}>0$, so that $\gamma\left(T_{2}-T_{\mathrm{c}}\right)$ will again become greater than $x(c)$, and $\frac{\partial T_{\mathrm{c}}}{\partial \gamma}$ will remain non-negative.

In order to prove part b), we focus on the first-order necessary equation for the local maxima of the profit function (49). We can re-write this equation as

$$
\exp \left(-\gamma\left(T_{2}-T\right)\right)=\frac{1}{c}\left(\frac{h(T)}{\gamma+h(T)}\right)
$$

where

$$
h(T)=\frac{d \log \left(M_{1}(T)\right)}{d T} .
$$


On the one hand, for $T>0$ we use (55) to obtain

$$
\begin{aligned}
h(T) & =\frac{1}{M_{1}} \frac{\left(1+\frac{q}{p}\right)^{\alpha_{1}+\alpha_{2}-1}}{\left(1+\frac{q \bar{D}}{p m}\right)^{\alpha_{1}+\alpha_{2}}}\left(1-\beta+\frac{q\left(1-\alpha_{1}\right) \bar{D}}{p m}\right) \frac{d \bar{D}(T)}{d T} \\
& \geq \frac{p(1-\beta)}{m(p+q)} \frac{d \bar{D}(T)}{d T} \geq \frac{p^{2} \beta(1-\beta)}{(p+q)} \min \left(1, \frac{\left(p \beta+q \alpha_{1}\right)^{2} e^{\left(p \beta+q \alpha_{1}\right) T_{2}}}{\left(q \alpha_{1}+p \beta e^{\left(p \beta+q \alpha_{1}\right) T_{2}}\right)^{2}}\right)=h^{*}\left(T_{2}>\text { (01) }\right)
\end{aligned}
$$

where we have utilized the definition of $\bar{D}(T>0)=\bar{D}_{1}(T)(6)$. We note that $\frac{\left(p \beta+q \alpha_{1}\right)^{2} e^{\left(p \beta+q \alpha_{1}\right) T_{2}}}{\left(q \alpha_{1}+p \beta e^{\left(p \beta+q \alpha_{1}\right) T_{2}}\right)^{2}}<$ 1 if and only if $T_{2}>\frac{2}{p \beta+q \alpha_{1}} \log \left(\frac{q \alpha_{1}}{p \beta}\right)$.

For $T<0$ (product 2 is introduced before product 1 ), we can introduce $\bar{T}=-T=$ $T_{1}-T_{2}>0$, so that

$$
\begin{aligned}
h(T) & =\frac{1}{M_{1}} \frac{d M_{1}}{d T}=\frac{d M_{2}}{d \bar{D}(\bar{T})} \frac{d \bar{D}(\bar{T})}{d \bar{T}} \frac{1}{m-M_{2}} \\
& =\frac{d M_{2}}{d \bar{D}(\bar{T})}\left(p(1-\beta)+q \alpha_{2} \frac{\bar{D}(\bar{T})}{m}\right) \frac{(m-\bar{D}(\bar{T}))}{\left(m-M_{2}\right)}
\end{aligned}
$$

where we have used the Bass identity $\frac{d \bar{D}(\bar{T})}{d \bar{T}}=\left(p(1-\beta)+q \alpha_{2} \frac{\bar{D}(\bar{T})}{m}\right)(m-\bar{D}(\bar{T}))$. Now, since for $\bar{T}>0, m \geq M_{2} \geq \bar{D}(\bar{T})$, we have $\frac{(m-\bar{D}(\bar{T}))}{\left(m-M_{2}\right)} \geq 1$, and

$$
h(T) \geq \frac{d M_{2}}{d \bar{D}(\bar{T})}\left(p(1-\beta)+q \alpha_{2} \frac{\bar{D}(\bar{T})}{m}\right) \geq \frac{p^{2} \beta(1-\beta)}{(p+q)}
$$

where we have used (55) and the fact that $0 \leq \bar{D}(\bar{T}) \leq m$. If $T_{2}<0$, and the product 2 is already on the market, the launch gap $T$ can only be negative and (59) has no solutions, provided that $\frac{1}{c}\left(\frac{h^{*}\left(T_{2}<0\right)}{\gamma+h^{*}\left(T_{2}<0\right)}\right)>1$, which is equivalent to $h^{*}\left(T_{2}<0\right)=\frac{p^{2} \beta(1-\beta)}{(p+q)}>\frac{c \gamma}{1-c}$. If, on the other hand, $T_{2} \geq 0$, then $T$ can be both positive as well as negative. Negative solutions to (59) do not exist if $\frac{p^{2} \beta(1-\beta)}{(p+q)}>\frac{c \gamma}{1-c}$, and the positive solutions are eliminated when $h^{*}\left(T_{2}>0\right)>\frac{c \gamma}{1-c}$.

\section{Proof of Theorem 4a}

Considering the case with $T_{1}^{*}=T_{2}^{*}$ we note that there are two possibilities: $T_{1}^{*}=T_{2}^{*}=0$ and $T_{1}^{*}=T_{2}^{*}>0$. The first case is possible if $\left.\frac{\partial \Pi_{1}\left(T_{1}, 0\right)}{\partial T_{1}}\right|_{T_{1}=0} \leq 0$ and $\left.\frac{\partial \Pi_{2}\left(0, T_{2}\right)}{\partial T_{2}}\right|_{T_{2}=0} \leq 0$. Using problem symmetry, we get

$$
\left.\frac{\partial \Pi_{1}\left(T_{1}, 0\right)}{\partial T_{1}}\right|_{T_{1}=0}=\left.\frac{\partial \Pi_{2}\left(0, T_{2}\right)}{\partial T_{2}}\right|_{T_{2}=0}=c \gamma \frac{m}{2}-(1-c) \frac{m}{2}\left(\frac{p\left(1+\frac{q}{p}\right)^{2 \alpha-1}}{2}\right)
$$

so that

$$
\left.\frac{\partial \Pi_{1}\left(T_{1}, 0\right)}{\partial T_{1}}\right|_{T_{1}=0}=\left.\frac{\partial \Pi_{2}\left(0, T_{2}\right)}{\partial T_{2}}\right|_{T_{2}=0} \leq 0 \Leftrightarrow c \leq c^{*} .
$$


The necessary condition for the existence of $T_{1}^{*}=T_{2}^{*}=T>0$ can be formulated as $\left.\frac{\partial \Pi_{2}\left(T, T_{2}\right)}{\partial T_{2}}\right|_{T_{2}=T}=0$, or

$$
\left.\frac{\partial \Pi_{2}\left(T, T_{2}\right)}{\partial T_{2}}\right|_{T_{2}=T}=c \gamma e^{-\gamma T} \frac{m}{2}-\left(1-c e^{-\gamma T}\right) \frac{m}{2}\left(\frac{p\left(1+\frac{q}{p}\right)^{2 \alpha-1}}{2}\right)=0,
$$

which, in turn, implies the unique solution

$$
T=\frac{1}{\gamma} \log \left(\frac{c}{c^{*}}\right) .
$$

The last expression is positive if and only if $c>c^{*}$. It is easy to check that the sufficient condition for (67) to be the local maximum of $\Pi_{2}\left(T, T_{2}\right),\left.\frac{\partial^{2} \Pi_{2}\left(T, T_{2}\right)}{\left(\partial T_{2}\right)^{2}}\right|_{T_{2}=T} \leq 0$, is satisfied. Thus, if entry-time Nash equilibrium exists, it is reached at (67). However, (67) may not be the global maximum of $\Pi_{2}\left(T, T_{2}\right)$ with respect to $T_{2}$ - for example, when $\Pi_{2}(T, 0)$ is greater than $\Pi_{2}(T, T)$. Thus, if $\Pi_{2}(T, 0)>\Pi_{2}(T, T)$, no solution to (20) and (21) exists. We note that

$$
\Pi_{2}(T, T)=\left(m-M_{1}(0)\right)\left(1-c e^{-\gamma T}\right)=\frac{m}{2}\left(1-c^{*}\right),
$$

while

$$
\Pi_{2}(T, 0)=\left(m-M_{1}(-T)\right)(1-c)=\left(\frac{m}{2}+\frac{D^{*}}{2}\left(\frac{1+\frac{q}{p}}{1+\frac{q D^{*}}{p m}}\right)^{2 \alpha-1}\right)(1-c),
$$

where

$$
D^{*}=m\left(1-\frac{q \alpha+\frac{p}{2}}{q \alpha+\frac{p}{2} e^{\left(q \alpha+\frac{p}{2}\right) T}}\right)=m\left(1-\frac{q \alpha+\frac{p}{2}}{q \alpha+\frac{p}{2}\left(\frac{c}{c^{*}}\right)^{\frac{q \alpha+\frac{p}{2}}{\gamma}}}\right) .
$$

Thus, $\Pi_{2}(T, 0)>\Pi_{2}(T, T)$ is equivalent to

$$
\frac{\left(1-c^{*}\right)}{(1-c)}<1+B(c)\left(\frac{1+\frac{q}{p}}{1+\frac{q}{p} B(c)}\right)^{2 \alpha-1} .
$$

where

$$
B(c)=1-\frac{q \alpha+\frac{p}{2}}{q \alpha+\frac{p}{2}\left(\frac{c}{c^{*}}\right)^{\frac{q \alpha+\frac{p}{2}}{\gamma}}} .
$$

\section{Proof of Theorem $4 b$}

i) We observe that $T_{1}^{l}=T_{2}^{l}=0 \Leftrightarrow\left(\left.\frac{\partial \Pi_{1}\left(T_{1}, 0\right)}{\partial T_{1}}\right|_{T_{1}=0} \leq 0,\left.\frac{\partial \Pi_{2}\left(0, T_{2}\right)}{\partial T_{2}}\right|_{T_{2}=0} \leq 0\right)$. Note that, according to (18)

$$
\left.\frac{\partial \Pi_{1}\left(T_{1}, 0\right)}{\partial T_{1}}\right|_{T_{1}=0}=\left.\left(\frac{d M_{1}\left(-T_{1}\right)}{d T_{1}}\left(1-c_{1} e^{-\gamma_{1} T_{1}}\right)+M_{1}\left(-T_{1}\right) c_{1} \gamma_{1} e^{-\gamma_{1} T_{1}}\right)\right|_{T_{1}=0}
$$




$$
\begin{aligned}
= & -\left.\frac{d M_{1}(T)}{d T}\right|_{T=0}\left(1-c_{1}\right)+M_{1}(0) c_{1} \gamma_{1} \\
= & -p(1-\beta) m \beta\left(1+\frac{q}{p}\right)^{\alpha_{1}+\alpha_{2}-1}\left(1-c_{1}\right) \\
& +c_{1} \gamma_{1} m \\
& \times\left(\frac{1-\alpha_{2}}{2-\alpha_{1}-\alpha_{2}}+\frac{p\left(\beta-\frac{1-\alpha_{2}}{2-\alpha_{1}-\alpha_{2}}\right)}{q\left(\alpha_{1}+\alpha_{2}-1\right)}\left(\left(1+\frac{q}{p}\right)^{\alpha_{1}+\alpha_{2}-1}-1\right)\right) \\
= & m\left(1-c_{1}\right)\left(\frac{c_{1} \gamma_{1}}{1-c_{1}}-\theta_{1}\right) \\
& \times\left(\frac{1-\alpha_{2}}{2-\alpha_{1}-\alpha_{2}}+\frac{p\left(\beta-\frac{1-\alpha_{2}}{2-\alpha_{1}-\alpha_{2}}\right)}{q\left(\alpha_{1}+\alpha_{2}-1\right)}\left(\left(1+\frac{q}{p}\right)^{\alpha_{1}+\alpha_{2}-1}-1\right)\right)(73)
\end{aligned}
$$

and, similarly,

$$
\begin{aligned}
\left.\frac{\partial \Pi_{2}\left(0, T_{2}\right)}{\partial T_{2}}\right|_{T_{2}=0}= & -p(1-\beta) m \beta\left(1+\frac{q}{p}\right)^{\alpha_{1}+\alpha_{2}-1}\left(1-c_{2}\right) \\
& +c_{2} \gamma_{2} m \\
& \times\left(\frac{1-\alpha_{1}}{2-\alpha_{1}-\alpha_{2}}-\frac{p\left(\beta-\frac{1-\alpha_{2}}{2-\alpha_{1}-\alpha_{2}}\right)}{q\left(\alpha_{1}+\alpha_{2}-1\right)}\left(\left(1+\frac{q}{p}\right)^{\alpha_{1}+\alpha_{2}-1}-1\right)\right) \\
= & m\left(1-c_{2}\right)\left(\frac{c_{2} \gamma_{2}}{1-c_{2}}-\theta_{2}\right) \\
& \times\left(\frac{1-\alpha_{1}}{2-\alpha_{1}-\alpha_{2}}-\frac{p\left(\beta-\frac{1-\alpha_{2}}{2-\alpha_{1}-\alpha_{2}}\right)}{q\left(\alpha_{1}+\alpha_{2}-1\right)}\left(\left(1+\frac{q}{p}\right)^{\alpha_{1}+\alpha_{2}-1}-1\right)\right)(.
\end{aligned}
$$

It is easy to show that

$$
\begin{aligned}
& \frac{1-\alpha_{2}}{2-\alpha_{1}-\alpha_{2}}+\frac{p}{q\left(\alpha_{1}+\alpha_{2}-1\right)}\left(\beta-\frac{1-\alpha_{2}}{2-\alpha_{1}-\alpha_{2}}\right)\left(\left(1+\frac{q}{p}\right)^{\alpha_{1}+\alpha_{2}-1}-1\right) \geq 0, \\
& \frac{1-\alpha_{1}}{2-\alpha_{1}-\alpha_{2}}-\frac{p}{q\left(\alpha_{1}+\alpha_{2}-1\right)}\left(\beta-\frac{1-\alpha_{2}}{2-\alpha_{1}-\alpha_{2}}\right)\left(\left(1+\frac{q}{p}\right)^{\alpha_{1}+\alpha_{2}-1}-1\right) \geq 0,(75)
\end{aligned}
$$

provided that $p<q$, which is the standard assumption in Bass dynamics.

Thus, $\left(\left.\frac{\partial \Pi_{1}\left(T_{1}, 0\right)}{\partial T_{1}}\right|_{T_{1}=0} \leq 0,\left.\frac{\partial \Pi_{2}\left(0, T_{2}\right)}{\partial T_{2}}\right|_{T_{2}=0} \leq 0\right) \Leftrightarrow\left(\frac{c_{1} \gamma_{1}}{1-c_{1}} \leq \theta_{1}, \frac{c_{2} \gamma_{2}}{1-c_{2}} \leq \theta_{2}\right)$.

ii) Below we prove the statement for the case of $T_{1}^{l}=0, T_{2}^{l}>0$ since the statement for $T_{1}^{l}>0, T_{2}^{l}=0$ is obtained by simple interchange of indices and replacement of $M_{1}$ by $m-M_{1}$ and $\beta$ by $1-\beta$. Note that $\left(T_{1}^{l}=0, T_{2}^{l}>0\right) \Leftrightarrow$

$$
\begin{gathered}
\left(\left.\frac{\partial \Pi_{1}\left(T_{1}, T_{2}^{l}\right)}{\partial T_{1}}\right|_{T_{1}=0} \leq 0,\left.\frac{\partial \Pi_{2}\left(0, T_{2}\right)}{\partial T_{2}}\right|_{T_{2}=T_{2}^{l}}=0,\left.\frac{\partial^{2} \Pi_{2}\left(0, T_{2}\right)}{\left(\partial T_{2}\right)^{2}}\right|_{T_{2}=T_{2}^{l}} \leq 0\right) . \text { Since, according to (19), } \\
\frac{\partial \Pi_{2}(0, T)}{\partial T}=-\frac{d M_{1}(T)}{d T}\left(1-c_{2} e^{-\gamma_{2} T}\right)+(m-M(T)) c_{2} \gamma_{2} e^{-\gamma_{2} T}
\end{gathered}
$$


and

$\frac{\partial^{2} \Pi_{2}(0, T)}{(\partial T)^{2}}=-\frac{d^{2} M_{1}(T)}{d T^{2}}\left(1-c_{2} e^{-\gamma_{2} T}\right)-2 \frac{d M_{1}(T)}{d T} c_{2} \gamma_{2} e^{-\gamma_{2} T}-(m-M(T)) c_{2}\left(\gamma_{2}\right)^{2} e^{-\gamma_{2} T}$.

Defining by $\widehat{T}_{2}$ all $T$ for which the right-hand side of (76) is 0 and the right-hand side of (77) is non-positive, we get (28). Finally, according to (18),

$$
\left.\frac{\partial \Pi_{1}\left(T_{1}, T_{2}^{l}\right)}{\partial T_{1}}\right|_{T_{1}=0}=-\frac{d M_{1}\left(T_{2}^{l}\right)}{d T}\left(1-c_{1}\right)+M_{1}\left(T_{2}^{l}\right) c_{1} \gamma_{1}
$$

so that

$$
\left.\frac{\partial \Pi_{1}\left(T_{1}, T_{2}^{l}\right)}{\partial T_{1}}\right|_{T_{1}=0} \leq 0 \Leftrightarrow \frac{c_{1} \gamma_{1}}{1-c_{1}} \leq \frac{1}{M_{1}\left(T_{2}^{l}\right)} \frac{d M_{1}\left(T_{2}^{l}\right)}{d T}
$$

iii) Similarly to the proofs in parts a) and b), we observe that $\left(T_{1}^{l}>0, T_{2}^{l}>0\right) \Leftrightarrow$ $\left(\left.\frac{\partial \Pi_{2}\left(T_{1}^{l}, T_{2}\right)}{\partial T_{2}}\right|_{T_{2}=T_{2}^{l}}=0,\left.\frac{\partial^{2} \Pi_{2}\left(T_{1}^{l}, T_{2}\right)}{\left(\partial T_{2}\right)^{2}}\right|_{T_{2}=T_{2}^{l}} \leq 0,\left.\frac{\partial \Pi_{1}\left(T_{1}, T_{2}^{l}\right)}{\partial T_{1}}\right|_{T_{1}=T_{1}^{l}}=0,\left.\frac{\partial^{2} \Pi_{1}\left(T_{1}, T_{2}^{l}\right)}{\left(\partial T_{1}\right)^{2}}\right|_{T_{1}=T_{1}^{l}} \leq 0\right)$. Now,

$\left.\frac{\partial \Pi_{2}\left(T_{1}^{l}, T_{2}\right)}{\partial T_{2}}\right|_{T_{2}=T_{2}^{l}}=-\frac{d M_{1}\left(T_{2}^{l}-T_{1}^{l}\right)}{d T}\left(1-c_{2} e^{-\gamma_{2} T_{2}^{l}}\right)+\left(m-M_{1}\left(T_{2}^{l}-T_{1}^{l}\right)\right) c_{2} \gamma_{2} e^{-\gamma_{2} T_{2}^{l}}$, $\left.\frac{\partial \Pi_{1}\left(T_{1}, T_{2}^{l}\right)}{\partial T_{1}}\right|_{T_{1}=T_{1}^{l}}=-\frac{d M_{1}\left(T_{2}^{l}-T_{1}^{l}\right)}{d T}\left(1-c_{1} e^{-\gamma_{1} T_{1}^{l}}\right)+M_{1}\left(T_{2}^{l}-T_{1}^{l}\right) c_{1} \gamma_{1} e^{-\gamma_{1} T_{1}^{l}}$.

Equating the right-hand sides of (80) to 0 , and denoting $T^{l}=T_{2}^{l}-T_{1}^{l}$, we get

$$
\begin{aligned}
& T_{1}^{l}=-\frac{1}{\gamma_{1}} \log \left(\frac{\frac{1}{c_{1}} \frac{1}{M_{1}\left(T^{l}\right)} \frac{d M_{1}}{d T}\left(T^{l}\right)}{\gamma_{1}+\frac{1}{M_{1}\left(T^{l}\right)} \frac{d M_{1}}{d T}\left(T^{l}\right)}\right), \\
& T_{2}^{l}=-\frac{1}{\gamma_{2}} \log \left(\frac{\frac{1}{c_{2}} \frac{1}{\gamma_{2}+M_{1}\left(T^{l}\right)} \frac{d M_{1}}{d T}\left(T^{l}\right)}{m-M_{1}\left(T^{l}\right)} \frac{d M_{1}\left(T^{l}\right)}{d T}\right),
\end{aligned}
$$

so that

$$
T^{l}=\log \left(\left(\frac{\frac{1}{c_{1}} \frac{1}{M_{1}\left(T^{l}\right)} \frac{d M_{1}}{d T}\left(T^{l}\right)}{\gamma_{1}+\frac{1}{M_{1}\left(T^{l}\right)} \frac{d M_{1}}{d T}\left(T^{l}\right)}\right)^{\frac{1}{\gamma_{1}}}\left(\frac{\frac{1}{c_{2}} \frac{1}{m-M_{1}\left(T^{l}\right)} \frac{d M_{1}}{d T}\left(T^{l}\right)}{\gamma_{2}+\frac{1}{m-M_{1}\left(T^{l}\right)} \frac{d M_{1}}{d T}\left(T^{l}\right)}\right)^{-\frac{1}{\gamma_{2}}}\right)
$$

Further,

$$
\left.\frac{\partial^{2} \Pi_{2}\left(T_{1}^{l}, T_{2}\right)}{\left(\partial T_{2}\right)^{2}}\right|_{T_{2}=T_{2}^{l}}=-\frac{d^{2} M_{1}\left(T_{2}^{l}-T_{1}^{l}\right)}{d T^{2}}\left(1-c_{2} e^{-\gamma_{2} T_{2}^{l}}\right)-2 \frac{d M_{1}\left(T_{2}^{l}-T_{1}^{l}\right)}{d T} c_{2} \gamma_{2} e^{-\gamma_{2} T_{2}^{l}}
$$




$$
\begin{aligned}
& -\left(m-M_{1}\left(T_{2}^{l}-T_{1}^{l}\right)\right) c_{2}\left(\gamma_{2}\right)^{2} e^{-\gamma_{2} T_{2}^{l}} \\
\left.\frac{\partial^{2} \Pi_{1}\left(T_{1}, T_{2}^{l}\right)}{\left(\partial T_{1}\right)^{2}}\right|_{T_{1}=T_{1}^{l}}= & \frac{d^{2} M_{1}\left(T_{2}^{l}-T_{1}^{l}\right)}{d T^{2}}\left(1-c_{1} e^{-\gamma_{1} T_{1}^{l}}\right)-2 \frac{d M_{1}\left(T_{2}^{l}-T_{1}^{l}\right)}{d T} c_{1} \gamma_{1} e^{-\gamma_{1} T_{1}^{l}} \\
& -M_{1}\left(T_{2}^{l}-T_{1}^{l}\right) c_{1}\left(\gamma_{1}\right)^{2} e^{-\gamma_{1} T_{1}^{l}} .
\end{aligned}
$$

Combining (84) and (85) with (80), we get

$$
\begin{aligned}
& \left.\frac{\partial^{2} \Pi_{2}\left(T_{1}^{l}, T_{2}\right)}{\left(\partial T_{2}\right)^{2}}\right|_{T_{2}=T_{2}^{l}} \leq 0 \Leftrightarrow-\frac{d^{2} M_{1}\left(T_{2}^{l}-T_{1}^{l}\right)}{d T^{2}} \leq \gamma_{2} \frac{d M_{1}\left(T_{2}^{l}-T_{1}^{l}\right)}{d T} \frac{1+c_{2} e^{-\gamma_{2} T_{2}^{l}}}{1-c_{2} e^{-\gamma_{2} T_{2}^{l}}} \\
& \left.\frac{\partial^{2} \Pi_{1}\left(T_{1}, T_{2}^{l}\right)}{\left(\partial T_{1}\right)^{2}}\right|_{T_{1}=T_{1}^{l}} \leq 0 \Leftrightarrow \frac{d^{2} M_{1}\left(T_{2}^{l}-T_{1}^{l}\right)}{d T^{2}} \leq \gamma_{1} \frac{d M_{1}\left(T_{2}^{l}-T_{1}^{l}\right)}{d T} \frac{1+c_{1} e^{-\gamma_{1} T_{1}^{l}}}{1-c_{1} e^{-\gamma_{1} T_{1}^{l}}}
\end{aligned}
$$

Now, using (81) and (82), we obtain

$$
\begin{aligned}
\gamma_{2} \frac{1+c_{2} e^{-\gamma_{2} T_{2}^{l}}}{1-c_{2} e^{-\gamma_{2} T_{2}^{l}}} & =\gamma_{2}+\frac{2}{m-M_{1}\left(T^{l}\right)} \frac{d M_{1}}{d T}\left(T^{l}\right), \\
\gamma_{1} \frac{1+c_{1} e^{-\gamma_{1} T_{1}^{l}}}{1-c_{1} e^{-\gamma_{1} T_{1}^{l}}} & =\gamma_{1}+\frac{2}{M_{1}\left(T^{l}\right)} \frac{d M_{1}}{d T}\left(T^{l}\right) .
\end{aligned}
$$

Thus, the necessary and sufficient conditions for $T_{1}^{l}$ and $T_{2}^{l}$ to be 'matching' maxima of the profit functions are expressed by (29) and (30) with (31). 

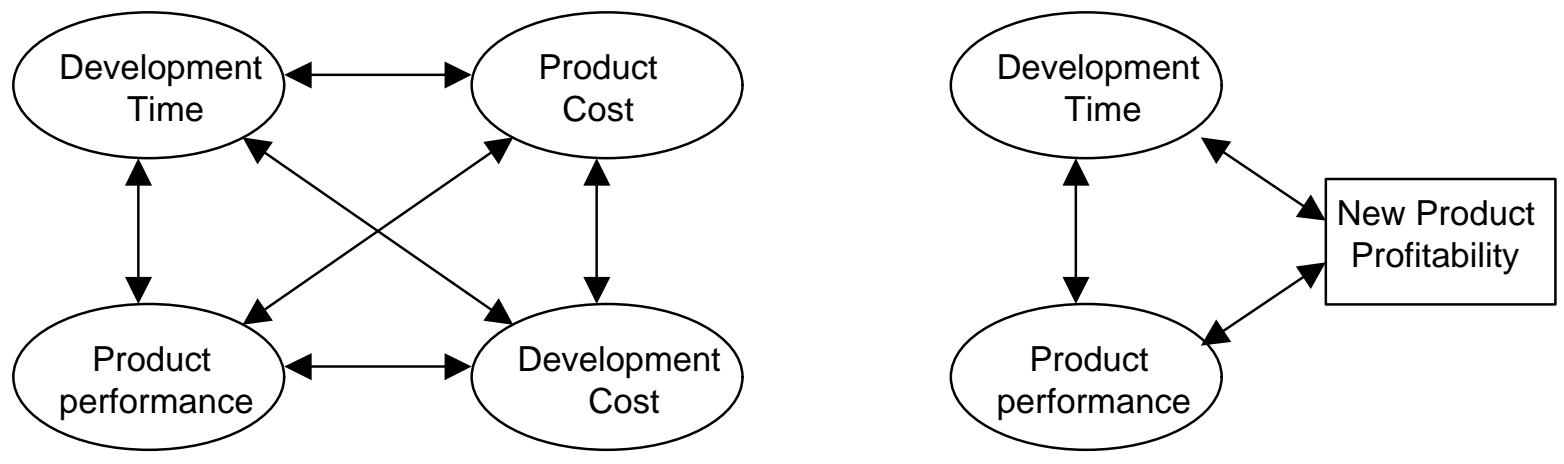

Source: Smith and Reinertsen (1991)

Ulrich and Eppinger (1999)

Figure 1: Trade-offs in product development (left) and the need for 'dollarizati

\begin{tabular}{|c|c|c|c|}
\hline & Methodology & Demand model & $\begin{array}{l}\text { Competitive activity } \\
\text { a) Number of competitors } \\
\text { b) Decision }\end{array}$ \\
\hline $\begin{array}{l}\text { Mahajan, Sharma, } \\
\text { Buzzell (1993) }\end{array}$ & Empirical & $\begin{array}{l}\text { Competitive diffusion with } \\
\text { a) saturation effect } \\
\text { b) word-of-mouth effect at } \\
\text { category and brand level } \\
\text { c) Brands can differ in their } \\
\text { word-of-mouth, reflecting } \\
\text { different attractiveness levels }\end{array}$ & NA \\
\hline $\begin{array}{l}\text { Krishnan, Bass, Kumar } \\
\text { (2000) }\end{array}$ & Empirical & $\begin{array}{l}\text { Competitive diffusion with } \\
\text { a) saturation effect } \\
\text { b) word-of-mouth effect at } \\
\text { category and brand level }\end{array}$ & NA \\
\hline $\begin{array}{l}\text { Eliashberg and Jeuland } \\
\text { (1986) }\end{array}$ & Normative & $\begin{array}{l}\text { Competitive diffusion with } \\
\text { a) saturation effect } \\
\text { b) NO word-of-mouth effect }\end{array}$ & $\begin{array}{l}\text { a) } 2 \text { (after entry) } \\
\text { b) Price for given entry }\end{array}$ \\
\hline $\begin{array}{l}\text { Fershtman, Mahajan, } \\
\text { and Muller (1990) }\end{array}$ & Normative & $\begin{array}{l}\text { Competitive diffusion with } \\
\text { a) NO saturation effect } \\
\text { b) Word-of-mouth effect at } \\
\text { the brand level }\end{array}$ & $\begin{array}{l}\text { a) } 2 \text { (after entry) } \\
\text { b) price and advertising for a } \\
\text { given entry }\end{array}$ \\
\hline $\begin{array}{l}\text { Kalish, Mahajan, and } \\
\text { Muller (1995) }\end{array}$ & Normative & $\begin{array}{l}\text { Competitive diffusion with } \\
\text { a) saturation effect } \\
\text { b) word-of-mouth effect only } \\
\text { at the brand level }\end{array}$ & $\begin{array}{l}\text { a) } 2 \text { (potential entrants in } \\
\text { second market } \\
\text { b) entry timing for second } \\
\text { market }\end{array}$ \\
\hline Present Manuscript & Normative & $\begin{array}{l}\text { Competitive diffusion with } \\
\text { a) saturation effect } \\
\text { b) word-of-mouth effect at } \\
\text { category and brand level } \\
\text { c) Brands can differ in their } \\
\text { word-of-mouth, reflecting } \\
\text { different attractiveness levels }\end{array}$ & $\begin{array}{l}\text { a) } 2 \text { (after entry) } \\
\text { b) timing of entry }\end{array}$ \\
\hline
\end{tabular}

Table 1: Empirical and Analytical Models of Competitive New Product Diffusion 


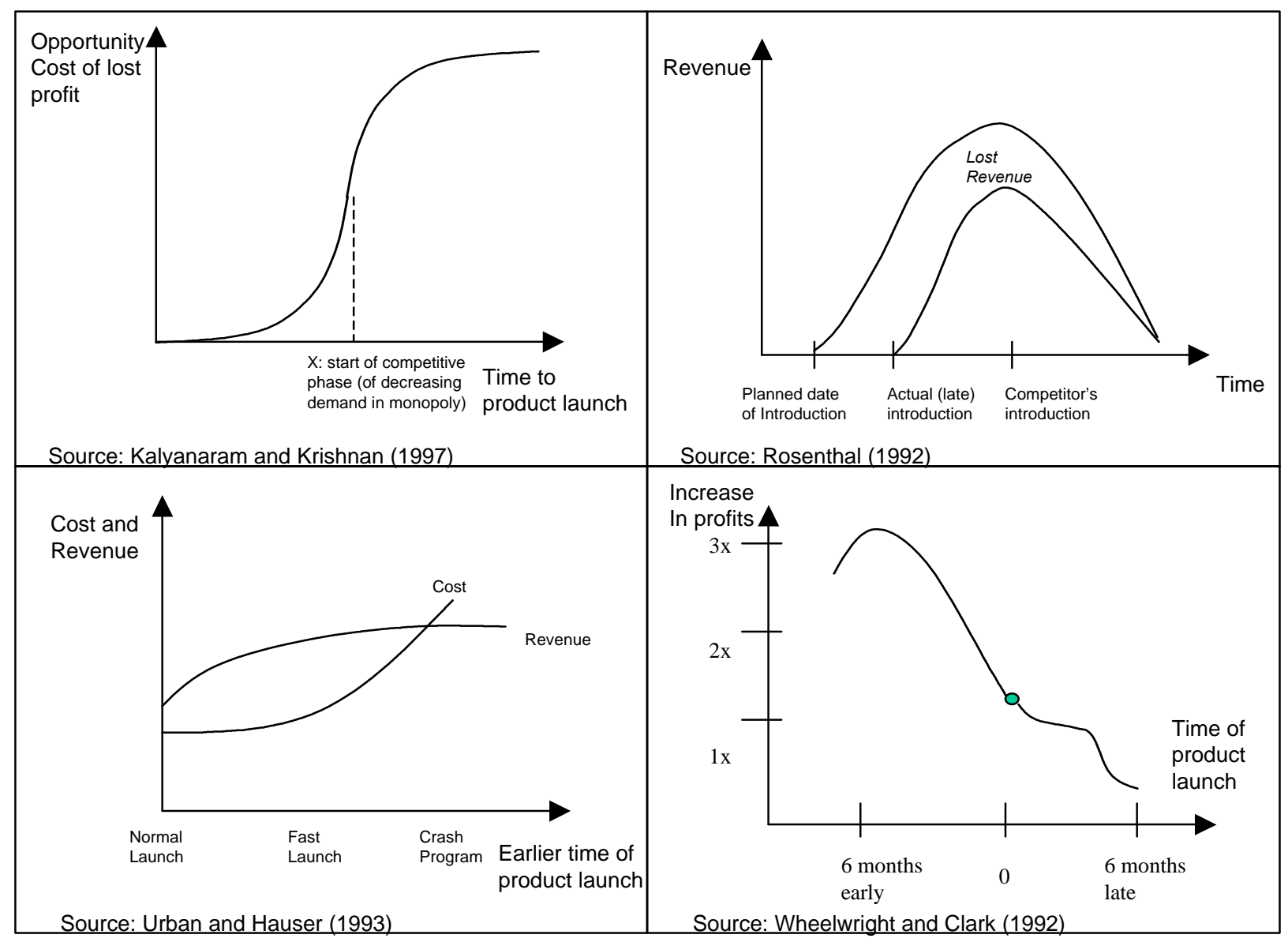

Figure 2: Qualitative arguments on the impact of an additional development time.

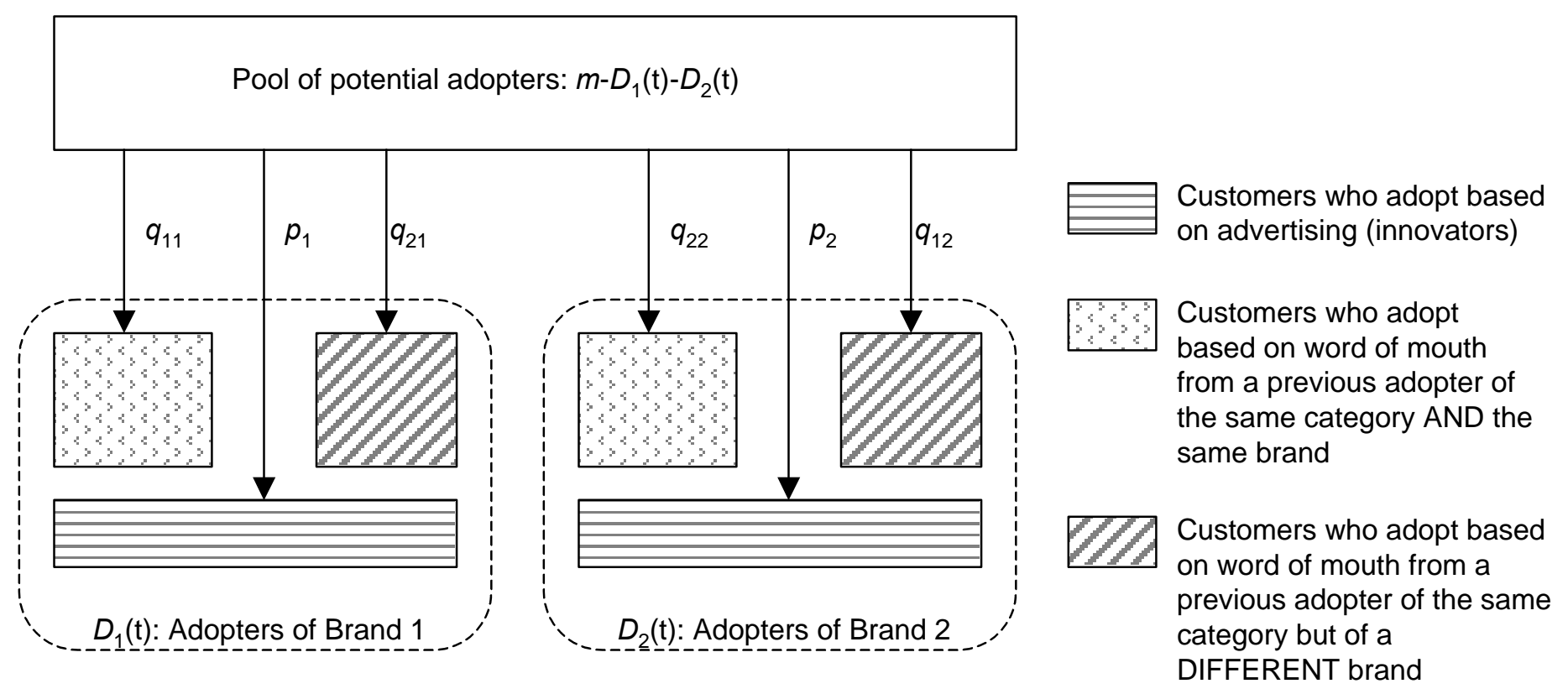

Figure 3: The model of brand level adoption in a presence of competition. 


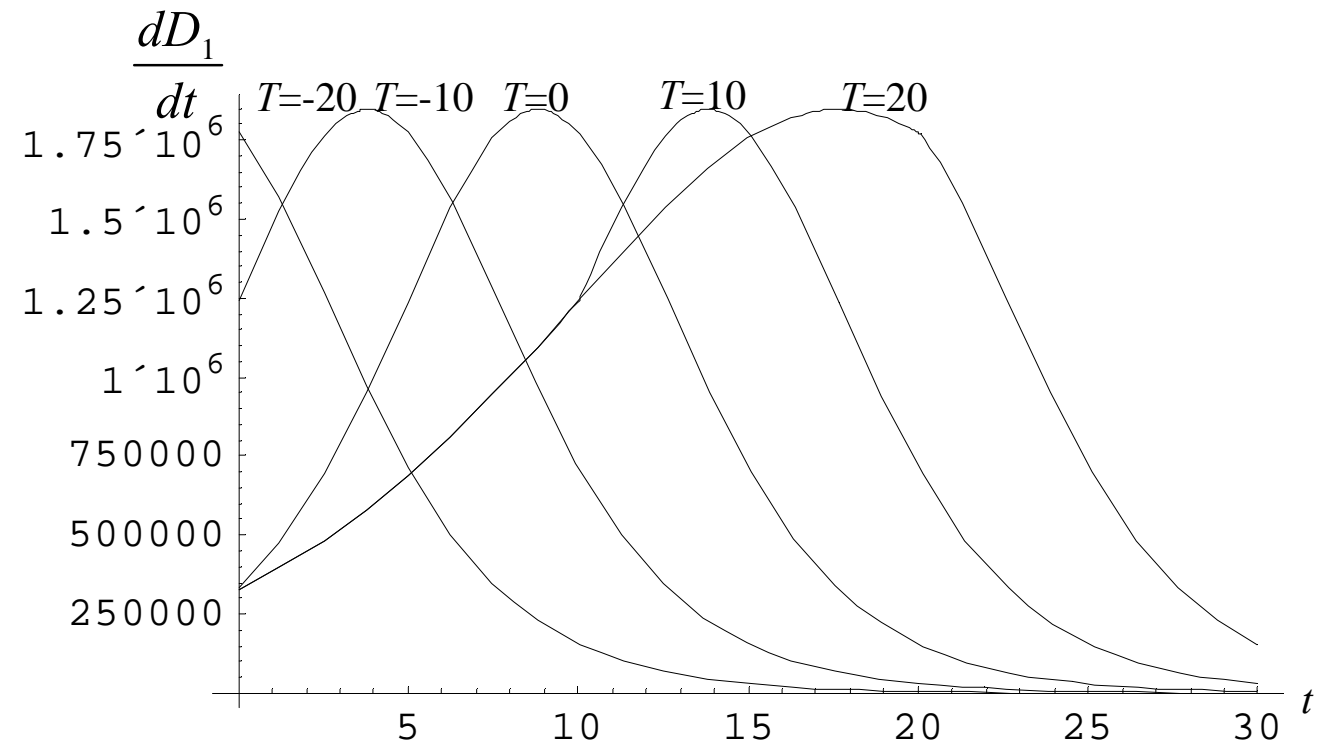

Figure 4: Demand rate for competitor 1 as a function of the launch gap $T(p=0.0163221, q=$ $0.325044, m=4.12984 \times 10^{7}, \alpha_{1}=\alpha_{2}=0.5, \beta=0.5$ ).

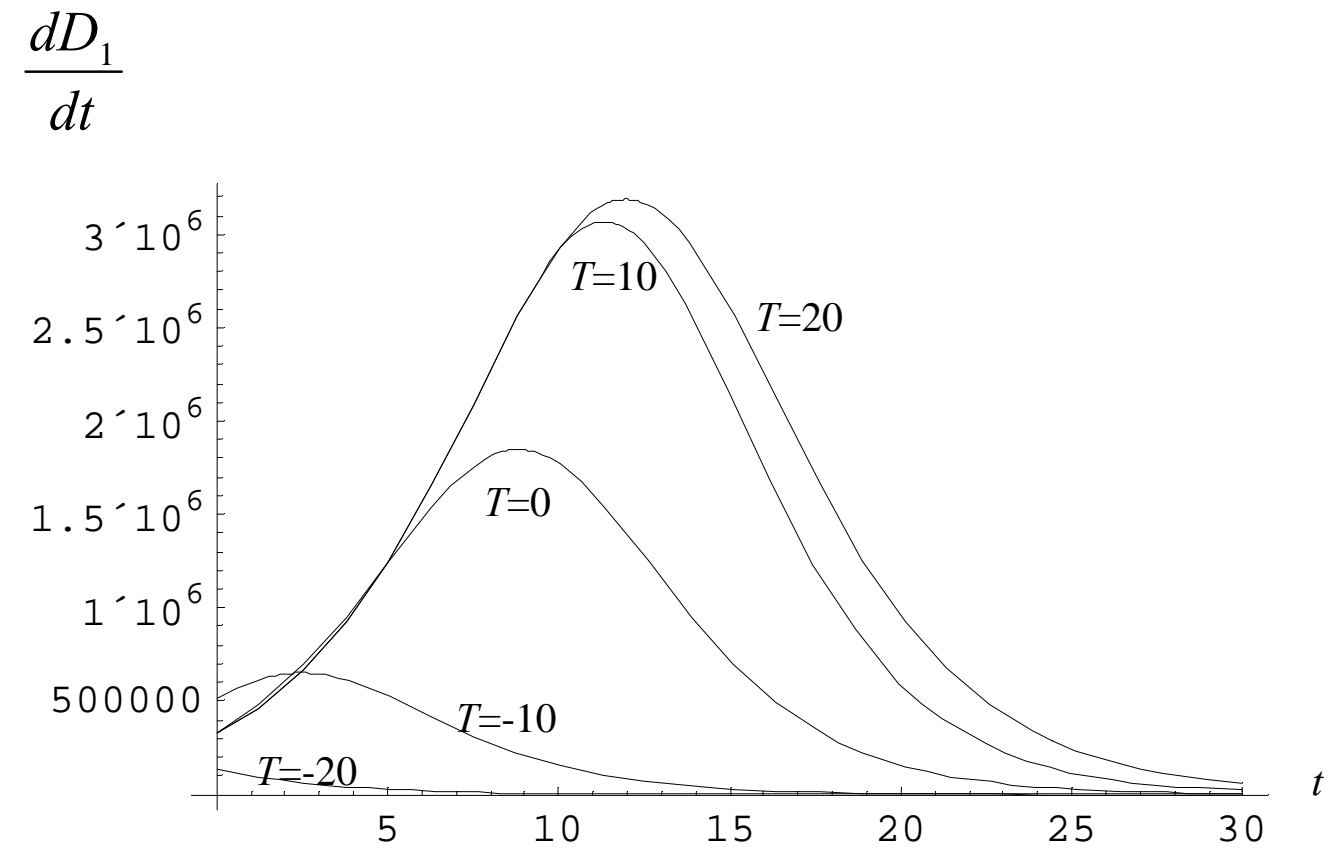

Figure 5: Demand rate for competitor 1 as a function of the launch gap $T(p=0.0163221, q=$ $0.325044, m=4.12984 \times 10^{7}, \alpha_{1}=\alpha_{2}=0.9, \beta=0.5$ ). 


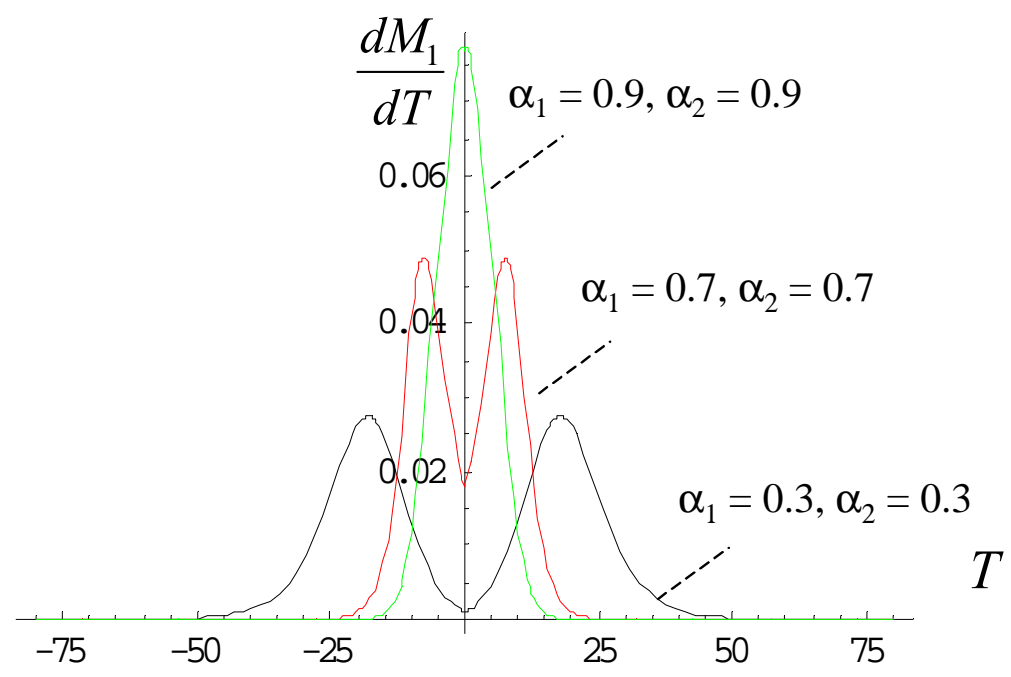

Figure 6: Rate of market share loss as a function of launch gap $T$

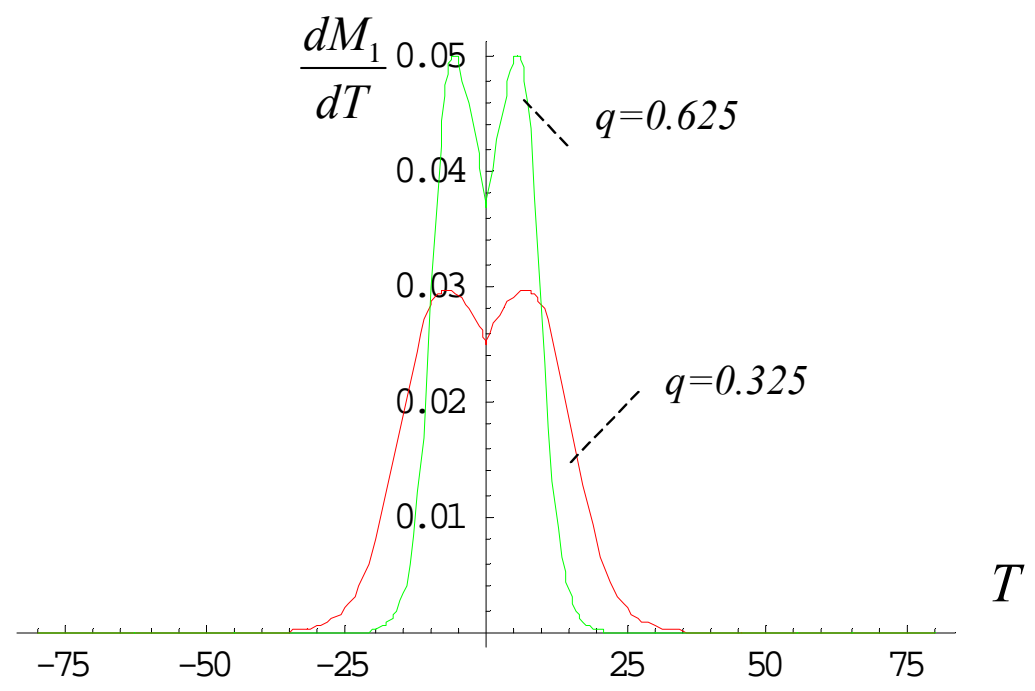

Figure 7: Rate of market share loss for different values of the category-level imitation parameter $q\left(c=0.2, \alpha_{1}=0.5, \alpha_{2}=0.5, \beta=0.5, T_{2}=50\right)$ 


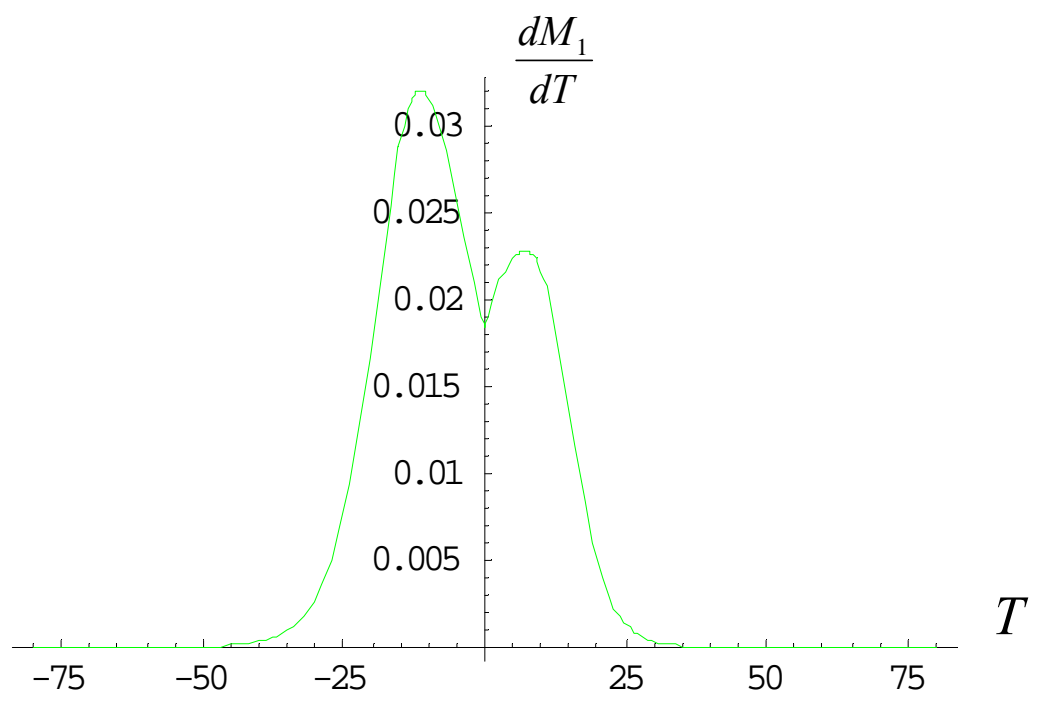

Figure 8: Rate of market share loss in the case of brands with different attractiveness levels $\left(c=0.2, \beta=0.5, T_{2}=50, \alpha_{1}=0.8, \alpha_{2}=0.6\right)$.

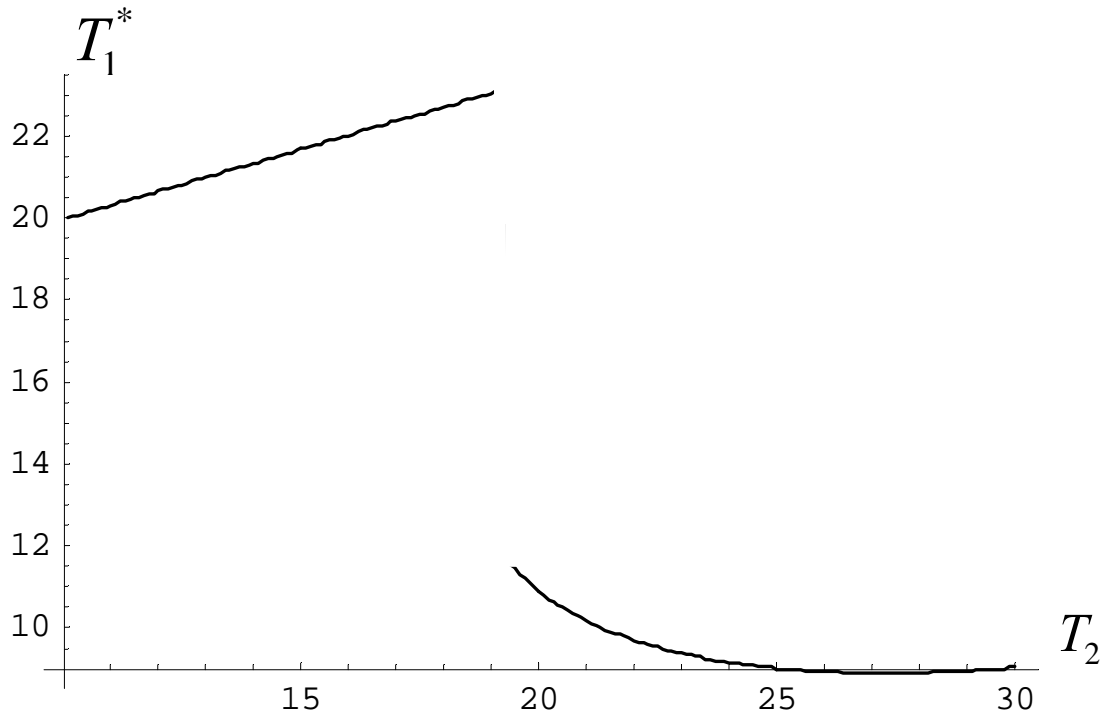

Figure 9: Optimal launch time for product $1, T_{1}{ }^{*}$, as a function of anticipated entry of product $2\left(c=0.6, \gamma=0.2, \alpha_{1}=\alpha_{2}=0.3, \beta=0.9\right)$. 

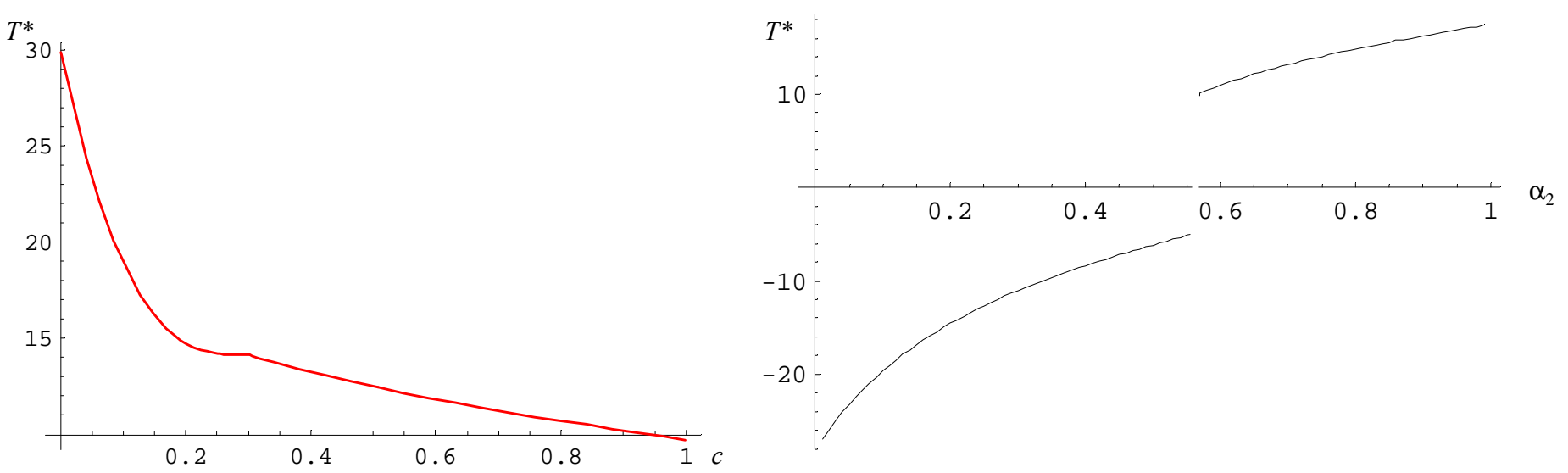

Figure 10ab: Sensitivity Analysis: optimal launch gap $T^{*}$ as a function of cost parameter $c(\gamma$ $\left.=0.1, \alpha_{1}=\alpha_{2}=0.9, \beta=0.5, T_{2}=30\right)(7 \mathrm{a})$ and attractiveness of competing product $\alpha_{2}\left(\gamma=0.1, \alpha_{1}=\right.$ $\left.0.09, c=0.99, \beta=0.9, T_{2}=30\right)(7 b)$.

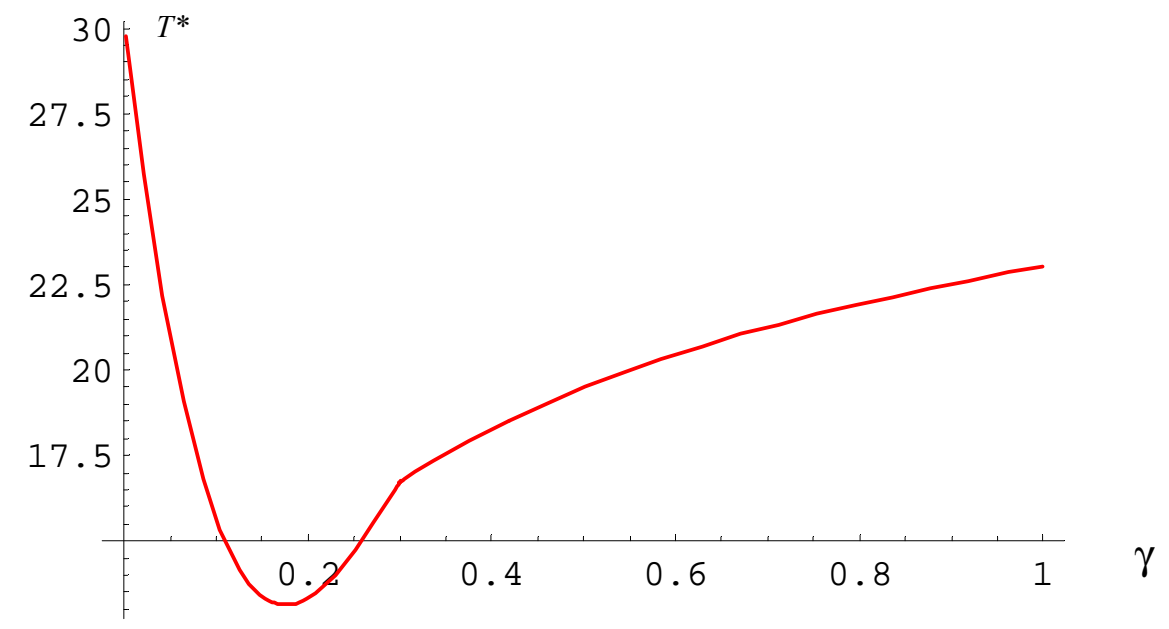

Figure 11: Optimal gap $T^{*}$ as a function of DFM-effectiveness parameter $\gamma$ $\left(c=0.6, \alpha_{1}=0.9, \alpha_{2}=0.9, \beta=0.5, T_{2}=30\right)$.

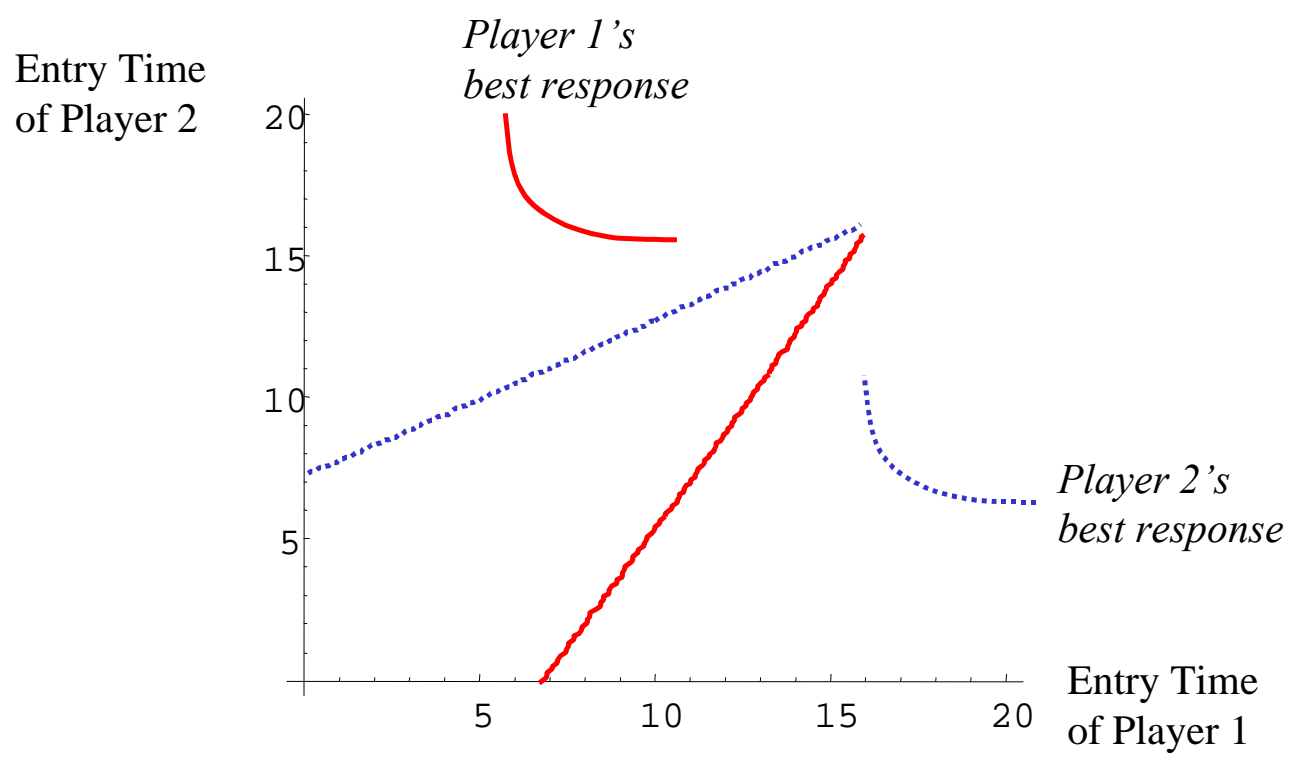

Figure 12: The best time response functions in the case of symmetric products $(p=0.0163221, q=$ $\left.0.325044, m=4.12984 \times 10^{7}, c=0.5, \gamma=0.1, \alpha_{1}=\alpha_{2}=0.55, \beta=0.5\right)$. 


\begin{tabular}{|l|l|l|l|}
\hline & $\beta=0.01$ & $\beta=0.5$ & $\beta=0.99$ \\
\hline$\alpha_{1}=\alpha_{2}=0.9$ & $T_{1}{ }^{*}=0 ; T_{2}{ }^{*}=4.06$ & $T_{1}{ }^{*}=0 ; T_{2}{ }^{*}=0$ & $T_{1}{ }^{*}=4.06 ; T_{2}{ }^{*}=0$ \\
\hline$\alpha_{1}=\alpha_{2}=0.55$ & No pure equilibrium & No pure equilibrium & No pure equilibrium \\
\hline$\alpha_{1}=0.9 ; \alpha_{2}=0.55$ & No pure equilibrium & $T_{1}{ }^{*}=0 ; T_{2}{ }^{*}=0$ & No pure equilibrium \\
\hline
\end{tabular}

Table 2: Existence of Nash-equilibrium entry times for two competitors with symmetric cost structures: $c_{1}=c_{2}=0.1, \gamma_{1}=\gamma_{2}=0.1\left(p=0.0163221, q=0.325044, m=4.12984 \times 10^{7}\right)$.

\begin{tabular}{|l|l|l|l|}
\hline & $\gamma_{1}=0.1 ; \gamma_{2}=0.1$ & $\gamma_{1}=0.1 ; \gamma_{2}=0.001$ & $\gamma_{1}=0.001 ; \gamma_{2}=0.1$ \\
\hline$c_{1}=c_{2}=0.5$ & $T_{1}{ }^{*}=T_{2}{ }^{*}=0.399$ & $T_{1}{ }^{*}=0.252 ; T_{2}{ }^{*}=0$ & $T_{1}{ }^{*}=0 ; T_{2}{ }^{*}=0.252$ \\
\hline$c_{1}=c_{2}=0.001$ & $T_{1}{ }^{*}=0 ; T_{2}{ }^{*}=0$ & $T_{1}{ }^{*}=0 ; T_{2}{ }^{*}=0$ & $T_{1}{ }^{*}=0 ; T_{2}{ }^{*}=0$ \\
\hline$c_{1}=0.5 ; c_{2}=0.001$ & $T_{1}{ }^{*}=0.252 ; T_{2}{ }^{*}=0$ & $T_{1}{ }^{*}=0.252 ; T_{2}{ }^{*}=0$ & $T_{1}{ }^{*}=0 ; T_{2}{ }^{*}=0$ \\
\hline
\end{tabular}

Table 3: Nash-equilibrium entry times for two products with symmetric diffusion parameters: $\alpha_{1}=\alpha_{2}=0.9, \beta=0.5\left(p=0.0163221, q=0.325044, m=4.12984 \times 10^{7}\right)$.

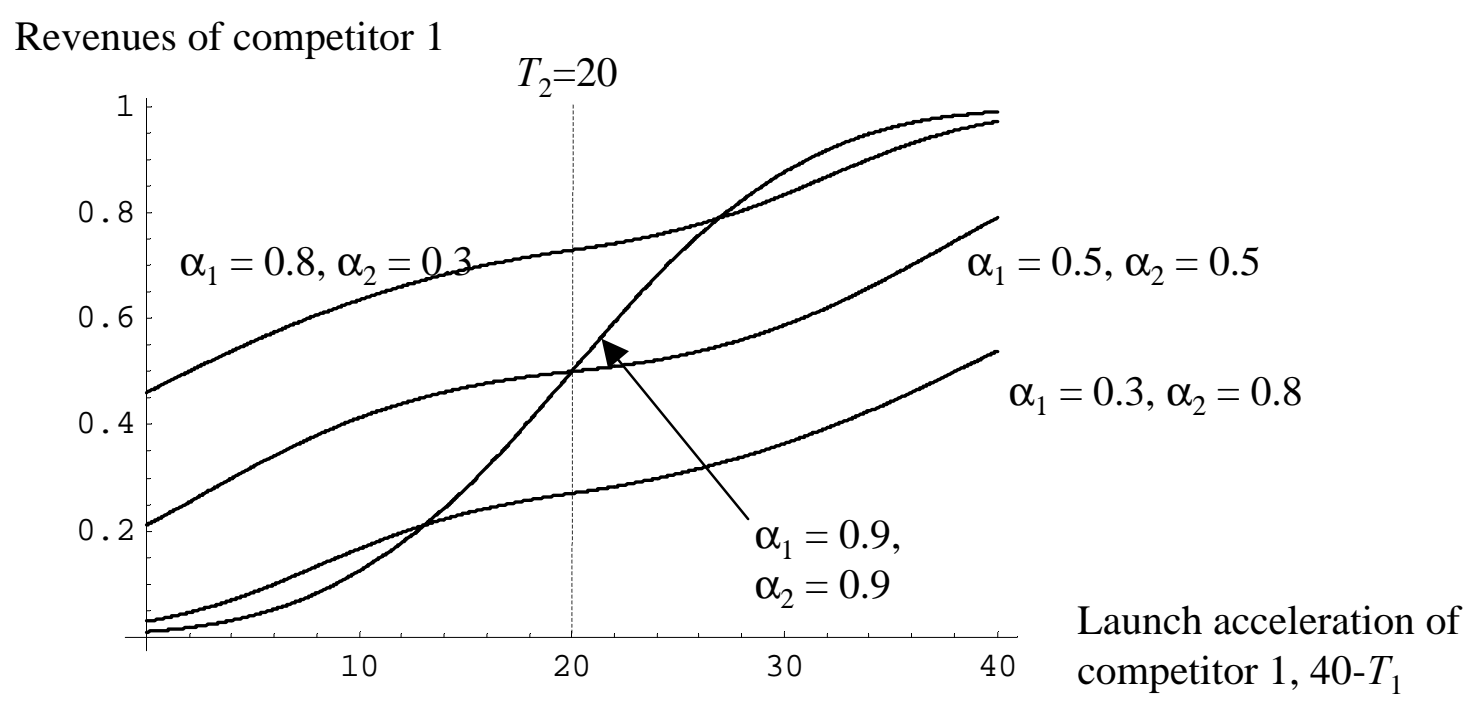

Figure 13: Revenues as a function of launch acceleration. 

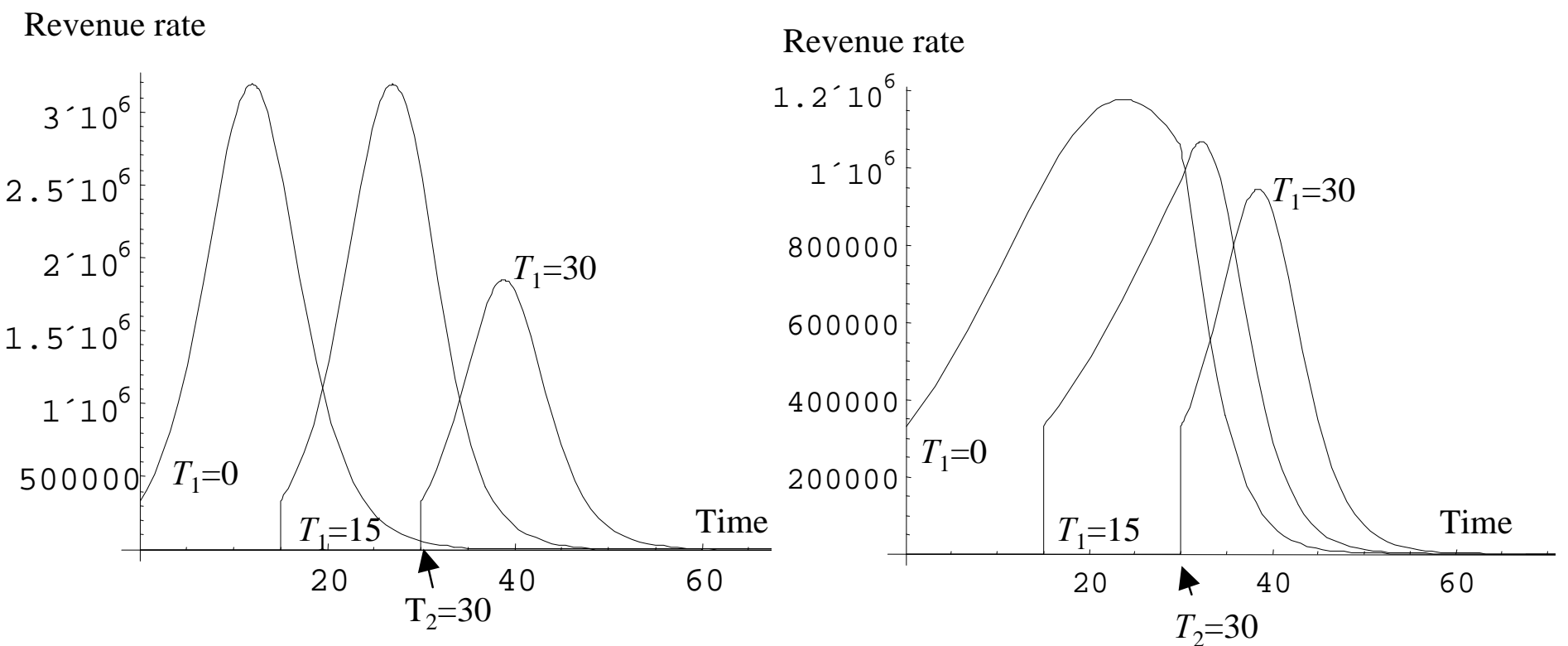

Figure 14ab: Revenue rate for different launch scenarios $\left(\alpha_{1}=0.9, \alpha_{2}=0.9\right.$ left and $\alpha_{1}=0.3, \alpha_{2}=0.8$ right .

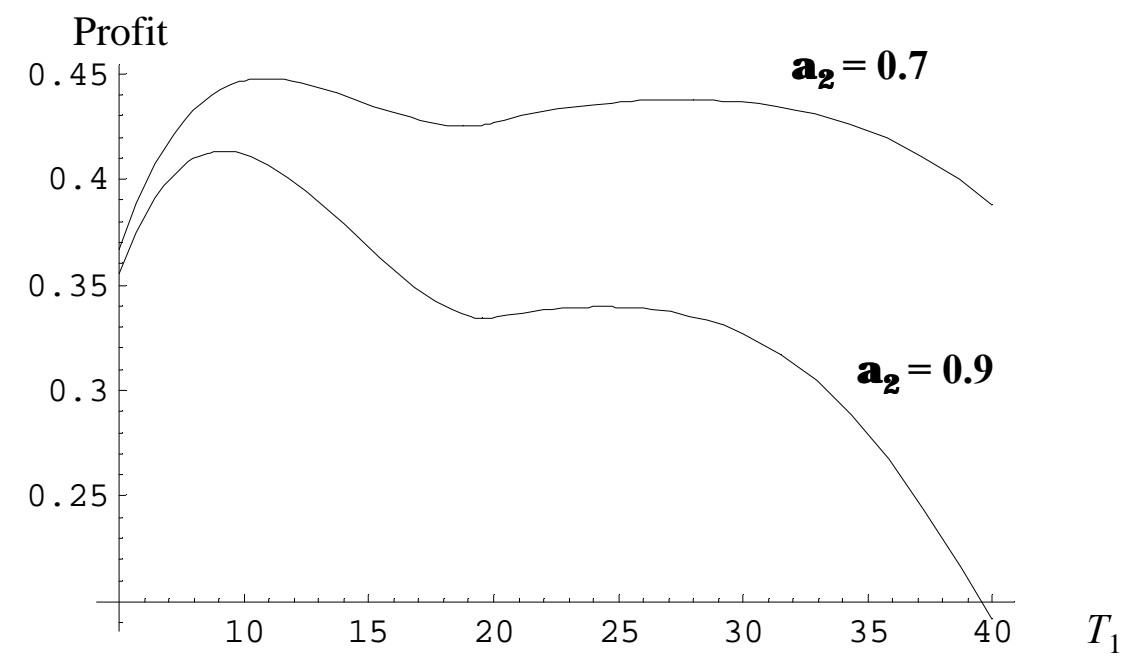

Figure 15: Local maxima in the profit function 